\title{
Comparative Analysis of Maleic Acid Copolymer-Based Lipid Nanodiscs Reveals Preferential Lipid and Protein Solubilization
}

Marta Barniol-Xicota, Steven Verhelst

Submitted date: 19/03/2020 - Posted date: 19/03/2020

Licence: CC BY-NC-ND 4.0

Citation information: Barniol-Xicota, Marta; Verhelst, Steven (2020): Comparative Analysis of Maleic Acid Copolymer-Based Lipid Nanodiscs Reveals Preferential Lipid and Protein Solubilization. ChemRxiv. Preprint. https://doi.org/10.26434/chemrxiv.12005175.v1

Membrane proteins are key in a large number of physiological and pathological processes. Their study often involves a prior detergent solubilization step, which strips away the membrane and can jeopardize membrane protein integrity. A recent alternative to detergents encompasses maleic acid based copolymers (xMAs), which disrupt the lipid bilayer and form lipid protein nanodiscs (xMALPs) soluble in aqueous buffer. Although xMALPs are often referred to as native nanodiscs, little is known about the resemblance of their lipid and protein content to the native bilayer. Here we have analyzed prokaryotic and eukaryotic XMALPs using lipidomics and in-gel analysis. Our results show that the XMALPs content varies with the chemical properties of the used XMA and that some of these nanodiscs are less native than initially thought.

File list (2)

Barniol-ChemRxiv-2020.pdf (1.36 MiB) view on ChemRxiv • download file 
Comparative analysis of maleic acid copolymer-based lipid nanodiscs reveals preferential lipid and protein solubilization

Marta Barniol-Xicota ${ }^{a}$ and Steven H. L. Verhelst *a,b

a. KU Leuven, Department of Cellular and Molecular Medicine, Laboratory of Chemical Biology, Herestr. 49 box 802, 3000 Leuven, Belgium. E-mail: steven.verhelst@kuleuven.be b. Leibniz Institute for Analytical Sciences ISAS, e.V., Otto-Hahn-Str. 6b, 44227 Dortmund, Germany. 


\begin{abstract}
Membrane proteins are key in a large number of physiological and pathological processes. Their study often involves a prior detergent solubilization step, which strips away the membrane and can jeopardize membrane protein integrity. A recent alternative to detergents encompasses maleic acid based copolymers (xMAs), which disrupt the lipid bilayer and form lipid protein nanodiscs (xMALPs) soluble in aqueous buffer. Although xMALPs are often referred to as native nanodiscs, little is known about the resemblance of their lipid and protein content to the native bilayer. Here we have analyzed prokaryotic and eukaryotic XMALPs using lipidomics and in-gel analysis. Our results show that the xMALPs content varies with the chemical properties of the used XMA and that some of these nanodiscs are less native than initially thought.
\end{abstract}




\section{Introduction}

Membrane proteins (MPs) are essential players in a wide variety of essential physiological processes. Certain specific MPs have been linked to various human diseases. For example, the membrane-bound kinase epidermal growth factor receptor plays a role in different types of cancer and malfunctioning of the ion channel $N$-MethylD-Aspartate receptor contributes to Alzheimer's Disease pathophysiology. Overall, MPs form two thirds of the total druggable targets in the cell. ${ }^{1,2}$ Unfortunately, MPs are less well understood than their soluble counterparts, because of their more difficult expression and purification procedures. ${ }^{3}$

Purification of MPs requires a prior solubilization step. Traditionally, this has been achieved with detergents, which isolate MPs in micelles by destroying the lipid bilayer. This de-lipidation can compromise structural integrity and may cause problems with protein activity or stability.

A decade ago, styrene maleic acid (SMA) co-polymers were reported as nanodisc forming agents. SMAs can extract MPs with their lipid microenvironment, forming styrene maleic acid lipid particles (SMALPs) soluble in aqueous buffer. Hence, SMAs can fully by-pass the use of detergents. In addition to SMA, several other maleic acid co-polymers (xMAs) have been synthesized as nanodisc forming agents (Figure1). These new xMAs have improved chemical properties or incorporate chemical modifications that broaden the downstream applications of xMALPs. For example, diisobutylene maleic acid (DIBMA) has aliphatic alkyl chains instead of aromatic groups, avoiding UV absorption and allowing the use of optical spectroscopic techniques as circular dichroism (CD). Another example is SMA-SH $\mathrm{SH}^{4}$ wich structure contains thiol groups that can act as chemical handles for reaction with fluorophores or other functional moieties.

Since their introduction, xMALPs have resulted in various exciting findings. For example, they enabled the structure determination of the Alternative Complex III (ACIII) 
by cryogenic electron microscopy (cryoEM) $)^{5}$ and the elucidation of naturally occurring oligomeric states of various MPs. ${ }^{6}$

In our previous work, we found XMALPs to stabilize fragile rhomboid proteases that self-process when in detergent micelles. ${ }^{7}$ In addition, rhomboid activity resembled more that in the membrane when in xMALPs than in when in micelles. However, we observed that the activity differed depending on the chemical properties of the xMA used. This finding made us question to what extent XMA nanodiscs resemble the native membrane and what lipids are present in the XMALPs. Only a few studies have analyzed the lipid content of SMALPs, focussing on phospholipids (PLs). Hence, the available information on the lipid content of XMALPs derived from biological membranes is very limited and it is unclear whether the different $\mathrm{xMAs}$ show preferential solubilization of certain protein species or lipid classes.

Here, we made a detailed comparison of chemically diverse xMA polymers in their solubilization properties of prokaryotic and eukaryotic membranes. In particular, we quantitatively and qualitatively compared the protein solubilization and performed MS-based lipidomics to identify the lipid composition. We found that xMAs display preferential solubilization of proteins and lipids depending on their chemical structure. This study has broad implications for future study of MPs in xMALPs, may help selecting xMAs with desired properties and aid future development of new xMAs with improved characteristics.

\section{Results}

A range of maleic acid based copolymers (xMAs) are currently available as nanodisc forming agents, with styrene maleic acid (SMA) as the most often used polymer. ${ }^{8}$ To date, unmodified SMA and diisobutylene maleic acid (DIBMA) are the only agents proven to directly extract and solubilize proteins from their native membrane. Here, in order to compare polymers, we selected SMA(3:1), SMA(2.3:1), SMA-QA and DIBMA (Figure 1), as these represent different chemical features. Whereas DIBMA is completely aliphatic, SMA contain aromatic groups derived from the styrene monomers which show strong UV absorption. In order to compare 
the effect of the styrene versus maleic acid ratio we chose for SMAs with ratios 3:1 and 2.3:1. Lastly, in order to assess the effect of charge, we included SMA-QA, which bears a positively charged quaternary ammonium ion instead of the two negative charges from the maleic acid moieties. In this study, we solubilized prokaryotic and eukaryotic membranes using the four abovementioned $x$ MAs as well as n-dodecyl- $\beta$-D-maltoside (DDM), a mild, non-ionic detergent that is often applied in solubilization of membrane proteins. Specifically, we used membrane pellets of $E$. coli, one of the most utilized species for recombinant protein expression, and of Jurkat cells, representing a cell line from human origin. In order to eliminate the influence on our experiments from culturing and lysing cells in different batches, we prepared one large batch of membrane pellets from each cell type, which was subsequently used in all solubilization studies.

\subsection{XMAs are efficient solubilizing agents for mammalian and bacterial membranes}

Since the first report that SMA co-polymers solubilize membrane proteins, ${ }^{9}$ their use has tremendously grown ${ }^{10}$ because of the attractive property of preserving annular lipids around the solubilized membrane protein. Obviously, the efficiency of a solubilization method is an important aspect to consider. We therefore compared the protein solubilization efficiencies of the selected co-polymers and DDM, for prokaryotic and eukaryotic membranes, by measurement of the protein concentration using a standard colorimetric assay. For E.coli membranes DDM is the most efficient method with $70 \%$ of total protein being solubilized and although there is a significantly lower efficiency for the XMA polymers, all but SMA-QA still solubilize over $50 \%$ of total membrane proteins (Figure $2 \mathrm{~A}$ ). This trend changed in case of the solubilization of Jurkat membrane proteins, where the xMAs did not have significantly lower efficiencies than DDM. The only exception was SMA-QA which was considerably less efficient than the detergent reference, but still solubilized approximately two thirds of all membrane proteins (Figure 2A).

\subsection{Different xMALPs show distinct patterns in total membrane protein solubilization}

One of the most attractive characteristics of the xMALPs is the possibility to directly solubilize membrane proteins from the lipid bilayer into lipid nanodiscs: the extracted 
proteins are never removed from their native membrane environment. For this reason, XMALPs are often called native nanodiscs. ${ }^{10-13}$ In light of the observed differences in the efficiencies of protein solubilization (see last paragraph), we next investigated the differences in protein content. SDS-PAGE analysis allowed for a qualitative comparison of the protein content in the XMALPs and DDM micelles with the original membrane pellet (Figure 2B). In order to prevent excessive smearing in SDS-PAGE, the polymer was largely removed from the proteins by chloroform/methanol protein precipitation. ${ }^{14}$ Overall, most proteins that occur in the E. coli membrane sample, are extracted into the lipid nanodiscs, except by SMA(2.3:1), which seems less efficient for higher MW proteins. This trend extends to all polymers when solubilizing Jurkat membranes, where high MW proteins seem to be less efficiently extracted. In addition, some xMAs seem to preferentially solubilize certain proteins in comparison with other xMAs (see, for example, star-indicated bands at $75 \mathrm{kDa}$ in the SMA-QA lane and at 18, 30 and $34 \mathrm{kDa}$ in the SMA(3:1) lane of Figure 2B). Surprisingly, all nanodiscs are unable to solubilize an E. coli protein appearing as an intense gel band in the $35 \mathrm{kDa}$ region.

For a first qualitative evaluation of the lipid contents of the nanodiscs, we analyzed the ratio of phosphate and protein. This ratio gives an indication of the total amount of phospholipids (PL) per protein amount. In general, the nanodiscs have a similar phosphate/protein ratio compared with the native membrane (Figure 2C). For E. coli, the nanodiscs show a slightly increased phosphate to protein ratio, which could indicate enrichment in PL in those nanodiscs. The observed trend does not occur when solubilizing Jurkat membranes.

\subsection{The PL headgroup composition of the soluble E. coli lipidome depends on the XMA used}

In light of the observed differences in lipid and protein content among the nanodiscs, we proceeded to analyze in detail their lipid composition.

For the analysis of E. coli membrane-derived samples, we used shotgun lipidomics with a method able to analyze PL and glycerolipids (GL). Unfortunately, the method used herein did not allow to measure cardiolipin (CL). Measuring PL, GL and CL 
simultaneously is far from trivial. ${ }^{15,16}$ Although some improved LC-MS/MS methods started to emerge in order to bridge this gap, these are not yet broadly implemented.

The GL content was always lower than $0.3 \%$, being the highest in the untreated membrane and significantly lower in DDM and the nanodiscs. Remarkably, no GLs were detected in SMA-QA nanodiscs.

PLs are the main lipid class in E. coli, therefore we analyzed in detail their composition characteristics for each of the solubilized fractions (see Figure 3A). The most abundant species was phosphatidylethanolamine (PE), being $81 \%$ of the total PLs present in the native membrane. This percentage was significantly reduced in the case of $\operatorname{SMA}(2.3: 1)(70 \%)$, DIBMA (63\%) and SMA-QA (68\%). The phosphatidylglycerol (PG) content was in all cases significantly increased (up to $21 \%$ PG content using $\operatorname{SMA}(2.3: 1))$ compared with the native membrane (12\% PG). No clear trend was observed for phosphatidylcholine (PC) which content was decreased in DDM (to $2 \%$ ), increased in the SMA-QA (to 9\%) and DIBMA (to 10\%) nanodiscs and similar to that in the membrane for unmodified SMAs (7\%). Other PLs, namely phosphatidylinositol (PI), phosphatidylserine (PS) and phosphatidic acid (PA) were only identified in trace amounts. The DDM solubilization afforded significantly decreased amounts of those lipids $(0.8 \%)$ compared to the membrane $(1.2 \%)$. On the contrary SMA-QA nanodiscs were enriched in PS, PA and PI (2.3\%).

Next, we investigated the fatty acid (FA) carbon chain length and saturation (Figure 3B-D). Regardless of the used solubilization method, saturated FA were depleted compared with the membrane; concurrently the percentage of unsaturated FA was increased (Figure 3B and supporting information FigureS1). In DDM and the nanodiscs formed by unmodified SMA, lipid species with 1 unsaturation were most increased compared with the membrane pellet. Interestingly, in DIBMA derived nanodiscs, the lipid species containing two unsaturations underwent the largest increase and were 2.3 times more abundant than in the membrane pellet. In the case of SMA-QA both 1 double bond $(\mathrm{db})$ and $2 \mathrm{db}$ species were increased in by $9 \%$ and $78 \%$ respectively. Note that 
E. coli lacks polyunsaturated fatty acids (PUFAs), therefore the lipids containing $2 \mathrm{dbs}$ comprise lipids with two monounsaturated fatty acid acyl chains.

In order to get insight in the chain length distribution, we determined the total number of carbons present per PL species and compared the different profiles with the membrane pellet (Figure 3C). For clarity, we refer to short (24-28 carbons), medium (29-32 carbons), long (3334 carbons) and very long (from 36 carbons) lipid species. All solubilizing agents except for SMA(3:1) showed less medium length species than the reference membrane. The content of long species was increased in DDM micelles. In the SMA(2.3:1) and SMA-QA the short and long species were more abundant than in the membrane. In the case of DIBMA the reduced presence of medium length species was compensated by an increased content of very long species (Figure 3C, green and red arrows). Remarkably, the $\operatorname{SMA}(3: 1)$ sample had no significant differences with the native membrane profile. The distinct distribution of FA chain lengths was also observed when analyzing specific PL headgroups (see Figure 3D for PE and supporting information Figure S2 for PC and PG). However, for some specific PL headgroups (e.g. PI), the chain length composition was virtually equal to the membrane (supporting information Figure S2).

\subsection{The composition of the soluble Jurkat lipidome varies for different XMALPs}

For the analysis of Jurkat membrane-derived samples we used shotgun lipidomics with a method able to analyze PLs, GLs and sphingolipids (SLs). Unfortunately, cholesterol esters could not be reliably measured using this method. As an alternative, we measured cholesterol using a separate fluorometric assay, the results of which are discussed later.

The membrane pellet was found to contain mainly PL (95\%) and small amounts of GL $(2.8 \%)$ and SL (1.9\%). The lipid composition of the DDM and the SMA-QA solubilized samples was practically identical to that of the native membrane, except for a minor increase in the GL content ( $3 \%$ and $5 \%$ of total lipids, respectively). In contrast, unmodified SMAs and DIBMA samples were depleted in PL which was compensated by an increase in GL. This general trend was more pronounced when using DIBMA 
(62\% PL, 35\% GL), followed by SMA(2.3:1) (71\% of PL, 27\% GL) and SMA(3:1) (87\% $P L, 10 \% \mathrm{GL})$.

Different classes of SL were identified but the SL composition and content did not experience significant variations among the samples, except for the SMA-QA sample in which the content of lactosylceramides was reduced compared to the membrane (Supporting Information Figure S3).

In all analysed samples we identified diacylglycerol (DG) and triacylglycerol (TG) species. Whereas in the membrane pellet TG:DG ratio was $1: 3$, in all solubilized samples the presence of TGs was decreased in favor of DGs. This ratio was approximately 1:7 when using DDM or SMA-QA and lowered to 1:32 for the unmodified SMAs or DIBMA (supporting information Figure S4).

The lipidomics analysis of PL showed that the membrane pellet contained 1.5\% PG, $38 \% \mathrm{PI}, 31 \% \mathrm{PC}$ and $29 \% \mathrm{PE}$. The same headgroup distribution was conserved in the lipidome solubilized by SMA-QA. Remarkably, the solubilization using unmodified SMAs showed a reduction of PG, PI and PE (up to 1.8, 3, and 3 fold respectively), in favor of PC which was 2.5 fold increased. This effect was more pronounced for $\operatorname{SMA}(2.3: 1)$ than for $\operatorname{SMA}(3: 1)$. A similar trend was observed when using DDM or DIBMA, but the differences compared with the membrane were less evident; for example, the DDM sample displayed a 1.5 fold increase in PC content (Figure 4B).

We observed that the detailed composition of the PC varied depending on the solubilization method used (supporting information Figure S5). In the membrane, the total PC fraction was composed of $88 \%$ intact PC and $12 \%$ PC of which the glycerol backbone had lost a fatty acid chain (lysoPC). The use of DDM showed a 3-fold increase in lysoPC mostly at the cost of PC and, to a minor degree, of ether lipids (PC$\mathrm{O}$ and $\mathrm{PC}-\mathrm{P}$ ). The detailed composition of PE remained more constant with the only change being the DIBMA PE-P content, which decreased to $10 \%$ compared to $21 \%$ found in the membrane (supporting information Figure S5). 


\section{The fatty acid saturation degree does not substantially affect xMALPs}

solubilization of Jurkat membranes. At this point, we inspected the degree of saturation in each sample. The global lipidome of the solubilized samples showed marked differences in the saturation degree, compared with the membrane (supporting information Figure S6). However, we hypothesized that the variation in the different lipid species between samples (see above) had an effect on total degree of saturation, provided that different lipid species display a distinct distribution of unsaturated FAs. To test this idea, we proceeded to analyze the saturation degree of SLs, GLs and each of the PL headgroups individually. Indeed, we observed that the changes in saturation profile compared with the membrane were very moderate for GLs and minimal for SLs and PL headgroups. In the GL fraction the unmodified xMA preferentially solubilized saturated species. Interestingly, all SMAs were enriched in GLs with an even number of double bonds. Strikingly, in the DIBMA fraction the monounsaturated species represented $62 \%$ of the total GL (compared with $19 \%$ in the membrane pellet). This increment is mainly due to a high abundance of the diacylglycerol (14:0/18:1) in the samples. The SL fraction remained virtually equal to the membrane for all $x M A$ solubilizing conditions, whereas DDM showed enrichment of certain unsaturated species (supporting information Figure S6). For the PL headgroups, the most noticeable changes were found for PC containing lipids in DDM and unmodified SMAs (Figure 5B). Some minor changes were seen for PE containing lipids solubilized with SMA(2.3:1), SMA-QA and DIBMA. Despite those, the xMAs mostly mimicked the membrane saturation degree for each PL headgroup (supporting information Figure S7).

The fatty acid chain length has little effect on xMA solubilization of Jurkat membranes. The FA chain length influences the thickness of the lipid bilayer. For this reason, we compared the number of carbons - as an estimate for the FA length - of the solubilized lipid species with those in the membrane. Similar to the unsaturation trends (see above), FA length profiles of the total lipid species looked dramatically different for different samples. We again analyzed lipid classes (SL, GL and the PL 
headgroups) separately in order test whether the differences are caused by the FA chain lengths themselves or by the distinct distribution of lipid species in the various xMA lipid nanodiscs.

For the FA length of SLs no major differences were observed between samples. As occurred for the unsaturations, the GLs displayed most differences in chain length. Here, two major aspects are worth highlighting: first, in all but the SMA-QA sample, there is a moderate to high reduction of lipids with 64 and more carbon atom chains. This observation matches with the reduction of TG species in favour of DGs (see above). Accordingly, the presence of shorter chain species is increased. Second, the DIBMA solubilized GLs show a 11.5-fold increase of the 32 carbon species compared with the membrane. This is mainly due to the increased presence of the diacylglycerol (14:0/18:1), previously highlighted as the cause for the increase of GL species with $1 \mathrm{db}$ (see above). The analysis of each PL headgroup species individually revealed that the carbon chain length profiles largely resemble that of the reference membrane (Figure 5 and supporting information Figure S8-9). The most remarkable difference is found for the DDM solubilized PC, which is 3-fold enriched in lysoPC (14-22 carbon species) with a consequential depletion in the rest of species.

2.5 Cholesterol content in anionic xMAs is membrane-like, but substantially altered in DDM micelles and SMA-QA nanodiscs

The cholesterol content was analyzed using the Amplex ${ }^{\mathrm{TM}}$ Red Cholesterol Assay Kit. The method of determination differs from the lipidomics used for the rest of the mammalian lipids, therefore the cholesterol results are not presented as percentage of total lipid content. The cholesterol content of unmodified SMAs and DIBMA resembled that in the membrane, being $42-46 \mathrm{nnmol}$ cholesterol/mg of protein versus $51 \mathrm{nnmol}$ cholesterol/mg of protein in the membrane. The solubilization with DDM was found to be 2.3 times enriched in cholesterol. On the contrary, barely any cholesterol was found in the SMA-QA sample, which content was more than $90 \%$ reduced compared with the membrane sample. 


\section{Discussion}

In this work, we compared the solubilization of native biological membranes by different membrane-solubilizing xMAs and DDM detergent. We showed that XMALP nanodiscs prepared with different XMA polymers display substantial quantitative and qualitative differences in their protein and lipid content. These differences occur in bacterial membranes (E. coli) as well as in mammalian membranes (Jurkat cell line).

\subsection{Protein solubilization efficiency of XMAs is adequate}

Our results show that the xMAs efficiently solubilize MPs from bacterial and mammalian membranes. In accordance with previous reports ${ }^{14}$ we found that DDM is slightly more efficient for solubilizing E. coli MPs than any of the tested xMAs. Remarkably, SMA-QA consistently presented the lowest efficiency. A computational study by Xue et al. suggested that the membrane disrupting properties of xMAs are linked to the stabilization of the xMALPs by interaction of the hydrophobic substituents with the bilayer interface while orienting their charged substituents to water-filled pores created by membrane defects. ${ }^{17}$ The lower overall charge of SMA-QA compared with other xMAs, might therefore explain its reduced solubilization properties. It will be interesting for future research to address this matter by comparing solubilization efficiencies of SMA-QA with different ratios of styrene to maleimide quaternary amines, which will provide distinctly charged SMA-QA polymers. Despite its lower efficiency the choice for SMA-QA over other XMAs may be justified because it can be used at acidic $\mathrm{pH}$ without precipitating, as is the case for other $\mathrm{xMAs}{ }^{18}$

\subsection{XMALP protein content varies for different XMAs}

Our experiments show that xMAs display preferential solubilization of MPs in both prokaroytic and eukaryotic membranes. This behavior has been observed in the past when using $\operatorname{SMA}(3: 1)$ and DIBMA with E. coli membranes. ${ }^{14,19,20}$ Of the solubilizing agents tested, DDM affords the most homogeneous solubilized protein content, probably due to its complete disruption of the lipid bilayer. Of the XMAs, DIBMA and SMA(3:1) showed the largest resemblance to the protein content present in the 
membrane, although some specific proteins as well as higher MW proteins seemed missing (Figure 2B). One may wonder what the cause of the observed differential solubilization could be. Although the inherent properties of a particular membrane protein, such as total charge or number of transmembrane domains, may have an impact, ${ }^{21,22}$ we do not exclude that the distinct lipid preferences of different xMAs (see below) will also affect the solubilization of proteins in specific lipid microenvironments. Future research on proteins with known localization in lipid domains will be needed to further address this question.

\section{3 xMALPs show distinct distribution of lipid species}

To ascertain whether xMAs preferentially solubilize certain lipids from biological membranes, we analyzed the lipid composition of the differently solubilized membranes. As we only detected PL species in the E. coli derived samples, we cannot comment on preferential solubilization of different lipid species from bacterial membranes. However, in the mammalian samples, we found differences in the solubilized lipid species. The relative abundance of GLs was markedly increased in $\operatorname{SMA}(3: 1), \operatorname{SMA}(2.3: 1)$ and DIBMA. A possible reason may be the hydrolysis of the PL headgroup, leading to conversion of a PL species into a GL species. Chemically, this is unlikely in view of the high stability of the phosphodiester bond. Enzymatically this process may be possible, if xMAs would superiorly solubilize phospholipase C. Another, probably more likely explanation may be the preferential solubilization of GLs over PLs by xMAs.

We did not observe considerable differences in the solubilization of SLs and cholesterol for most of the xMAs. However, within the PL species, there were distnct preferences of XMAs for PG (increased in E. coli samples) and PC (increased in Jurkat samples), whereas PE was generally disfavored. We wondered what may be the driving force between these observed preferences and considered several molecular characteristics. The overall charge does not seem to play a role, because the solubilization behaviour of PC and PE, which both have a zwitterionic headgroup and a 
net zero charge, is generally opposite of each other. Being uncharged also does not provide an explanation, as the uncharged GLs are increased, but cholesterol is not. Previous work with model membranes suggested that solubilization is not regulated by the properties of individual lipid constituents but by the overall physical state of the bilayer. ${ }^{23}$ The lipid bilayer is structured in ordered (Lo) and disordered regions (Ld). Some lipid species, such as cholesterol and SLs, are preferentially found in Lo regions. To date, there is no consensus on which phase is more prone to XMALP formation. ${ }^{23-26}$ Nevertheless, it is known that Lo regions are more sensitive than Ld regions to perturbations induced by surfactant insertion into the lipid bilayer. ${ }^{27}$ Hence, the fact that SL and cholesterol, found in Lo regions, are conserved in all unmodified SMA and DIBMA, points at more accurate Lo solubilization, in line with this hypothesis. Other lipid species, such as PLs and DGs, can be found in both phases depending on specific lipid properties (see next paragraph). The effect of DGs in the membrane packing is still controversial: they are reported by some to have a condensing effect, increasing the acyl chain order and bilayer thickness, ${ }^{28}$ but by others are seen as membrane order disruptors. ${ }^{29}$

\subsection{PL headgroup composition varies between xMALPs}

The properties of PLs largely depend on their headgroup. The headgroups not only confer distinct chemical properties such as charge and polarity, but also influence the overall lipid shape. The size balance between the headgroups and the FA defines the PL geometry: PLs with large headgroups such as PC or PG have a cylindrical geometry and tend to form flat membranes, whereas smaller headgroups give rise to a conical structure, leading to a curvature in the membrane. ${ }^{30}$ The observation in our study that PG (in E. coli membranes) and PC (in mammalian membranes) have higher occurrence in the xMA lipid nanodiscs, also supports the hypothesis that xMA polymers may disrupt highly structured regions of the membrane more easily. Specifically, an increase in the occurrence of these lipids in the xMA nanodiscs reflects that lipid species in more disordered regions are less well extracted and solubilized by xMAs. 


\subsection{Preferential lipid solubilization is linked to xMA chemical properties}

The comparative lipidomics analysis in this study shows that the specific $\mathrm{xMA}$ determines the composition of the soluble lipidome. Our observations are in contradiction with the extended idea that $\mathrm{xMAs}$ are promiscuous solubilizing agents which do not preferentially extract lipids from the membrane. This discrepancy could be explained by several factors. First of all, only a few studies have analyzed the lipid content of SMALPs derived from biological membranes. ${ }^{11,20,31-35}$ In these reports, the utilized SMA was $\operatorname{SMA}(2: 1)$, which is less hydrophobic than the SMAs we used here. The different chemical properties of our SMAs may account for the discrepancy with the results of the studies that conclude there is no preferential lipid extraction by $\operatorname{SMA}(2: 1)$, even though some point out the preferential solubilization of unsaturated FA chains by $\operatorname{SMA}(2: 1) \cdot{ }^{36}$ Second, a couple of studies using $\operatorname{SMA}(3: 1)$ reported non-preferential lipid extraction, but these have mainly used analysis by TLC and quantification by densitomety. ${ }^{35}$ The LC-MS analysis used here is more sensitive and may reveal differences less well detectable by TLC, especially when these changes are subtle. Third, SMA(3:1) showed clear preferential lipid solubilization in Jurkat membranes (see Figure4), but when applied to E. coli membranes, there were only minor differences with the composition of the membrane. This latter result is in line with previous studies that used LC-MS to study the lipid composition of $\operatorname{SMA}(3: 1)$ E. coli nanodiscs. ${ }^{20,34}$ The majority of studies towards lipid solubilization by xMAs, however, have used model membranes consisting of only two or three lipid species and lacking protein content. ${ }^{23-}$ ${ }^{25}$ In the absence of proteins, lipids behave differently than in biological membranes (e.g. distinct phase partitioning), ${ }^{30,37}$ suggesting that the $\mathrm{xMA}$ lipid preference in native membranes may not occur in model lipid bilayers. We here provide data that suggest that the overall membrane properties rather than the individual lipid species regulate xMA solubilization, underlining the importance of using biological membranes as study object. 


\section{Conclusions}

We have analyzed the composition of xMALPs derived from eukaryotic and prokaryotic membranes using LC-MS/MS and SDS-PAGE. The lipid and protein content varied between xMALPs and compared with the native membrane. The solubilization efficiency of the xMAs versus a widely used detergent was only slightly decreased for prokaryotic membranes and equally efficient for eukaryotic membranes. These results show that the differences in solubilization efficiency between xMAs and detergent do not prevent the choice for the co-polymers. In addition, the small decrease in the solubilization efficiency is counterbalanced by the reported increased protein stability that the xMAs offer in exchange..$^{5,7}$

The distinct protein solubilization observed here indicates that certain MPs may benefit from the use of a specific xMA. More detailed proteomics-based comparative studies will be necessary to answer the question why xMAs preferentially solubilize particular MPs and which factors govern these preferences. Until that question is resolved, it will be beneficial to test various xMAs when solubilizing and purifying a specific MP of interest. For now, our analysis reveals that xMAs have preferential protein and lipid solubilization characteristics that vary with the chemical structure of the applied xMA.

Here, we also provided evidence that the solubilization preferences are guided by the bulk of lipid species, but not by the FA composition (i.e. length and degree of saturation). This suggests that the shape of the lipid species or, more general, the degree of order in the structure of the membrane could be the determining factor for solubilization, whereas the membrane thickness or packing strength has a much milder effect. To get more insight into the detailed membrane features that control xMALP formation, complex models that mimic biological membranes will be necessary. Altogether, this may lead to the future design of novel polymers with improved membrane disruption properties or to chemically tuning them to extract specific membrane regions. 
The preferential lipid extraction reported here has important implications for future analysis of XMALP lipid contents and reveals the importance of the correct controls. For example, the use of XMALPs in the analysis of lipids that immediately surround MPs (annular lipid shell) may suffer from a bias stemming from the inherent preferences of the $\mathrm{xMA}$. This can be addressed by analyzing the lipid content of unpurified $\mathrm{xMA}$ nanodiscs as control. Another recommendation involves the analysis of the insoluble fraction after XMA solubilization: this will reveal whether the XMALPs with a MP of interest are representative for the total protein or only a small fraction.

Our initial question was to what extent xMA nanodiscs resemble the native membrane. This study revealed that the resemblance depends on the used xMA. Ideally, a more membrane-like environment would be preferred. From our results we conclude SMA-QA is the most membrane-like if looking only at lipid content and disregarding cholesterol. Nevertheless, when both protein and lipid content are taken into account, SMA(3:1) gives the most balanced solubilization.

Finally, this study has also shown that the differences in protein and lipid content in xMALPs are more subtle in E. coli than in Jurkat membranes. This make us wonder how membranes from other species such as yeast or extremophiles would behave with different $x$ MAs. Future studies detailing the xMA solubilization of such systems would help sketching a complete picture of xMA solubilization preferences. Some of our future efforts are along these lines and will be reported in due course.

\section{Acknowledgements}

We acknowledge Polyscope and BASF for kindly providing the SMA and DIBMA polymers. We thank Jef Callebaut for helping with matlab scripts for data analysis, Suravi Chakrabarty for help in cell culture and Jonas Dehairs for collecting lipidomics data of Jurkat samples. We acknowledge funding from Horizon2020 - Marie Curie fellowship ( $n^{\circ}$ 752252) to MBX, FWO for a post-doctoral fellowship ( $n^{\circ} 12$ Y0720N) to MBX, the Ministerium für Kultur und Wissenschaft des Landes Nordrhein-Westfalen, 
the Regierende Bürgermeister von Berlin-inkl. Wissenschaft und Forschung, and the Bundesministerium für Bildung und Forschung.

\section{Notes and references}

1 J. P. Overington, B. Al-Lazikani and A. L. Hopkins, Nat. Rev. Drug Discov., 2006, 5, 993-6.

2 R. Santos, O. Ursu, A. Gaulton, A. P. Bento, R. S. Donadi, C. G. Bologa, A. Karlsson, B. Al-Lazikani, A. Hersey, T. I. Oprea and J. P. Overington, Nat. Rev. Drug Discov., 2016, 16, 19-34.

3 J. G. Almeida, A. J. Preto, P. I. Koukos, A. M. J. J. Bonvin and I. S. Moreira, Biochim. Biophys. Acta - Biomembr., 2017, 1859, 2021-2039.

4 S. Lindhoud, V. Carvalho, J. W. Pronk and M. E. Aubin-Tam, Biomacromolecules, 2016, $17,1516-1522$.

5 C. Sun, S. Benlekbir, P. Venkatakrishnan, Y. Wang, S. Hong, J. Hosler, E. Tajkhorshid, J. L. Rubinstein and R. B. Gennis, Nature, 2018, 557, 123-126.

6 N. Hellwig, O. Peetz, Z. Ahdash, I. Tascón, P. J. Booth, V. Mikusevic, M. Diskowski, A. Politis, Y. Hellmich, I. Hänelt, E. Reading and N. Morgner, Chem. Commun., 2018, 54, 13702-13705.

7 M. Barniol-Xicota and S. H. L. Verhelst, J. Am. Chem. Soc., 2018, 140, 14557-14561.

8 Z. Stroud, S. C. L. Hall and T. R. Dafforn, Methods, 2018, 147, 106-117.

9 T. J. Knowles, R. Finka, C. Smith, Y. P. Lin, T. Dafforn and M. Overduin, J. Am. Chem. Soc., 2009, 131, 7484-7485.

M. Overduin and B. Klumperman, Eur. Polym. J., 2019, 110, 63-68.

11 J. M. Dörr, M. C. Koorengevel, M. Schäfer, A. V. Prokofyev, S. Scheidelaar, E. A. W. Van Der Cruijsen, T. R. Dafforn, M. Baldus and J. A. Killian, Proc. Natl. Acad. Sci. U. S. A., 2014, 111, 18607-18612.

12 M. Overduin and M. Esmaili, SLAS Discov., 2019, 24, 943-952.

13 S. Rehan, V. O. Paavilainen and V. Jaakola, BBA - Biomembr., 2017, 1859, 1059-1065. 
14 A. O. Oluwole, B. Danielczak, A. Meister, J. O. Babalola, C. Vargas and S. Keller, Angew. Chemie - Int. Ed., 2017, 56, 1919-1924.

D. Oursel, C. Loutelier-Bourhis, N. Oragne, S. Chevalier, V. Norris and C. M. Lange, Rapid Commun. Mass Spectrom., 2007, 21, 1721-1728.

T. A. Garrett, A. C. O’Neill and M. L. Hopson, Rapid Commun. Mass Spectrom., 2012, 26, 2267-2274.

17 M. Xue, L. Cheng, I. Faustino, W. Guo and S. J. Marrink, Biophys. J., 2018, 115, 494502.

18 T. Ravula, N. Z. Hardin, S. K. Ramadugu, S. J. Cox and A. Ramamoorthy, Angew. Chemie - Int. Ed., 2018, 57, 1342-1345.

19 A. H. Kopf, J. M. Dörr, M. C. Koorengevel, F. Antoniciello, H. Jahn and J. A. Killian, Biochim. Biophys. Acta - Biomembr., 2020, 1862, 183125.

20 I. Prabudiansyah, I. Kusters, A. Caforio and A. J. M. Driessen, Biochim. Biophys. Acta Biomembr., 2015, 1848, 2050-2056.

21 N. L. Lee, Sarah C; Pollock, Biochem. Soc. Trans., 2016, 44, 1011-1018.

22 S. C. Lee, T. J. Knowles, V. L. G. Postis, M. Jamshad, R. A. Parslow, Y. Lin, A. Goldman, P. Sridhar, M. Overduin, S. P. Muench and T. R. Dafforn, Nat. Protoc., 2016, 11, 11491162.

23 R. Cuevas Arenas, J. Klingler, C. Vargas and S. Keller, Nanoscale, 2016, 8, 1501615026.

24 J. J. Dominguez Pardo, J. M. Dörr, A. lyer, R. C. Cox, S. Scheidelaar, M. C. Koorengevel, V. Subramaniam and J. A. Killian, Eur. Biophys. J., 2017, 46, 91-101.

25 S. Scheidelaar, M. C. Koorengevel, J. D. Pardo, J. D. Meeldijk, E. Breukink and J. A. Killian, Biophys. J., 2015, 108, 279-290.

26 M. Jamshad, V. Grimard, I. Idini, T. J. Knowles, M. R. Dowle, N. Schofield, P. Sridhar, Y. Lin, R. Finka, M. Wheatley, O. R. T. Thomas, R. E. Palmer, M. Overduin, C. Govaerts, J. M. Ruysschaert, K. J. Edler and T. R. Dafforn, Nano Res., 2015, 8, 774-789.

27 H. Heerklotz, Q. Rev. Biophys., 2008, 41, 205-264. 
28 M. Alwarawrah, F. Hussain and J. Huang, Biochim. Biophys. Acta - Biomembr., 2016, 1858, 253-263.

29 P. Campomanes, V. Zoni and S. Vanni, Commun. Chem., 2019, 2, 1-8.

30 T. Harayama and H. Riezman, Nat. Rev. Mol. Cell Biol., 2018, 19, 281-296.

31 A. C. K. Teo, S. C. Lee, N. L. Pollock, Z. Stroud, S. Hall, A. Thakker, A. R. Pitt, T. R. Dafforn, C. M. Spickett and D. I. Roper, Sci. Rep., 2019, 9, 1-10.

32 E. Reading, Z. Hall, C. Martens, T. Haghighi, H. Findlay, Z. Ahdash, A. Politis and P. J. Booth, Angew. Chemie - Int. Ed., 2017, 56, 15654-15657.

33 S. Rehan, V. O. Paavilainen and V. P. Jaakola, Biochim. Biophys. Acta - Biomembr., 2017, 1859, 1059-1065.

34 V. Schmidt, M. Sidore, C. Bechara, J. P. Duneau and J. N. Sturgis, Biochim. Biophys. Acta - Biomembr., 2019, 1861, 431-440.

35 D. J. K. Swainsbury, S. Scheidelaar, N. Foster, R. van Grondelle, J. A. Killian and M. R. Jones, Biochim. Biophys. Acta - Biomembr., 2017, 1859, 2133-2143.

36 E. Reading, Z. Hall, C. Martens, T. Haghighi, H. Findlay, Z. Ahdash, A. Politis and P. J. Booth, Angew. Chemie - Int. Ed., 2017, 56, 15654-15657.

37 F. Schneider, D. Waithe, M. P. Clausen, S. Galiani, T. Koller, G. Ozhan, C. Eggeling and E. Sezgin, Mol. Biol. Cell, 2017, 28, 1507-1518. 


\section{Figures}

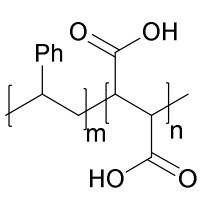

SMA

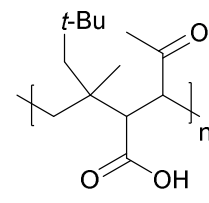

DIBMA

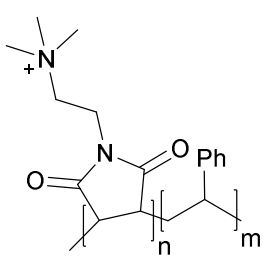

SMA-QA

Figure 1 Chemical formulas of the nanodisc forming polymers used in this work. Unmodified SMA: $m=2.3$ or $3, n=1$. DIBMA: $m=1, n=1$. SMA-QA: $m=3, n=1$.

A

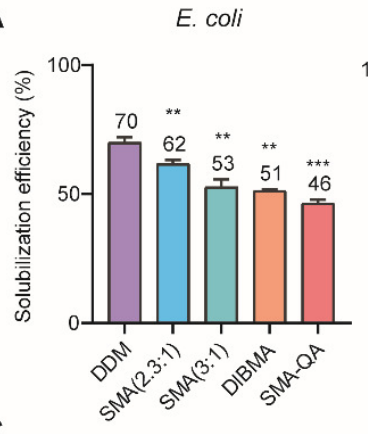

C

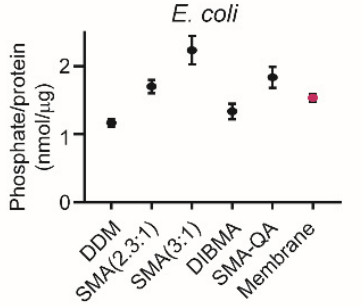

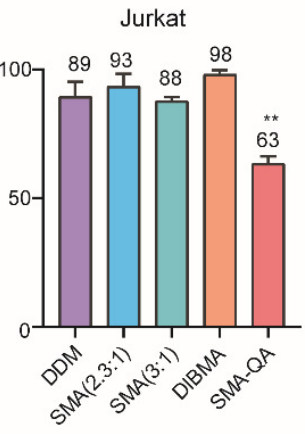

Jurkat

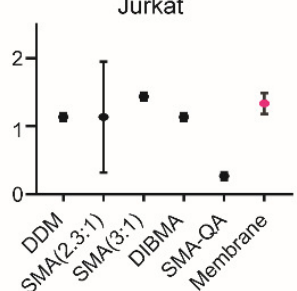

B

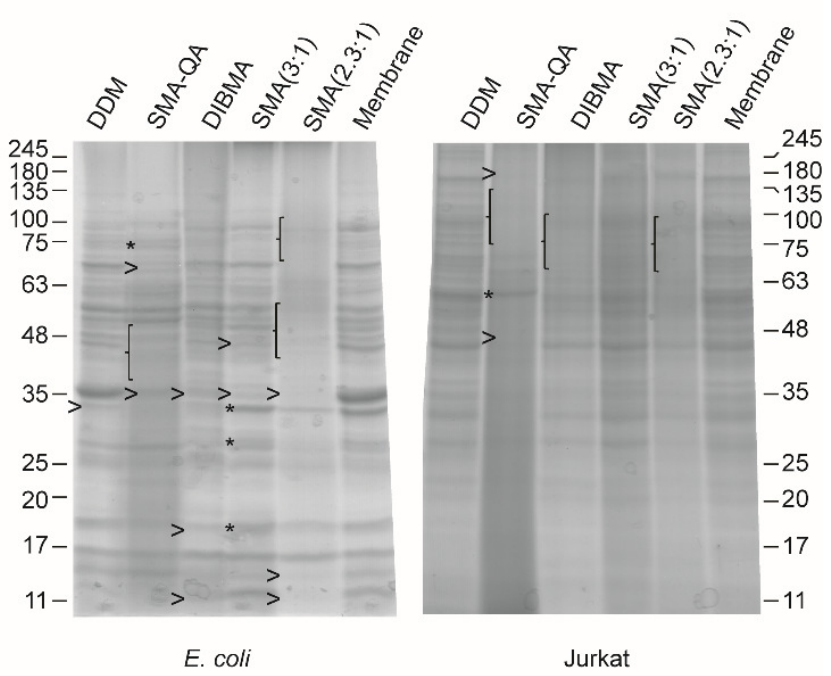

Figure 2 Solubilization of $E$. coli and Jurkat membrane pellets with DDM and different XMAs.

(A) Protein solubilization efficiency, as measured by BCA assay of soluble and insoluble fractions after solubilization with DDM or xMAs. Data correspond to three technical replicates. Error bars represent \pm S.D. Significant differences (student's t-test) are denoted as * $(p<0.05)$, ${ }^{* *}(p<0.01)$, or ${ }^{* * *}(p<0.001)$ (B) Coomassie stained gels of membrane protein samples solubilized by different XMAs or DDM. An arrowhead or accolade indicates proteins that are substantially lower abundant than in the membrane pellet. Stars indicate examples of protein bands solubilized by one XMA, but not or much worse by others. (C) Ratio of phosphate (measured by determining total inorganic phosphorus) and protein amount present in the soluble fractions or in the membrane, determined by BCA assay. Data points correspond to three technical replicates. Error bars represent \pm S.D. 

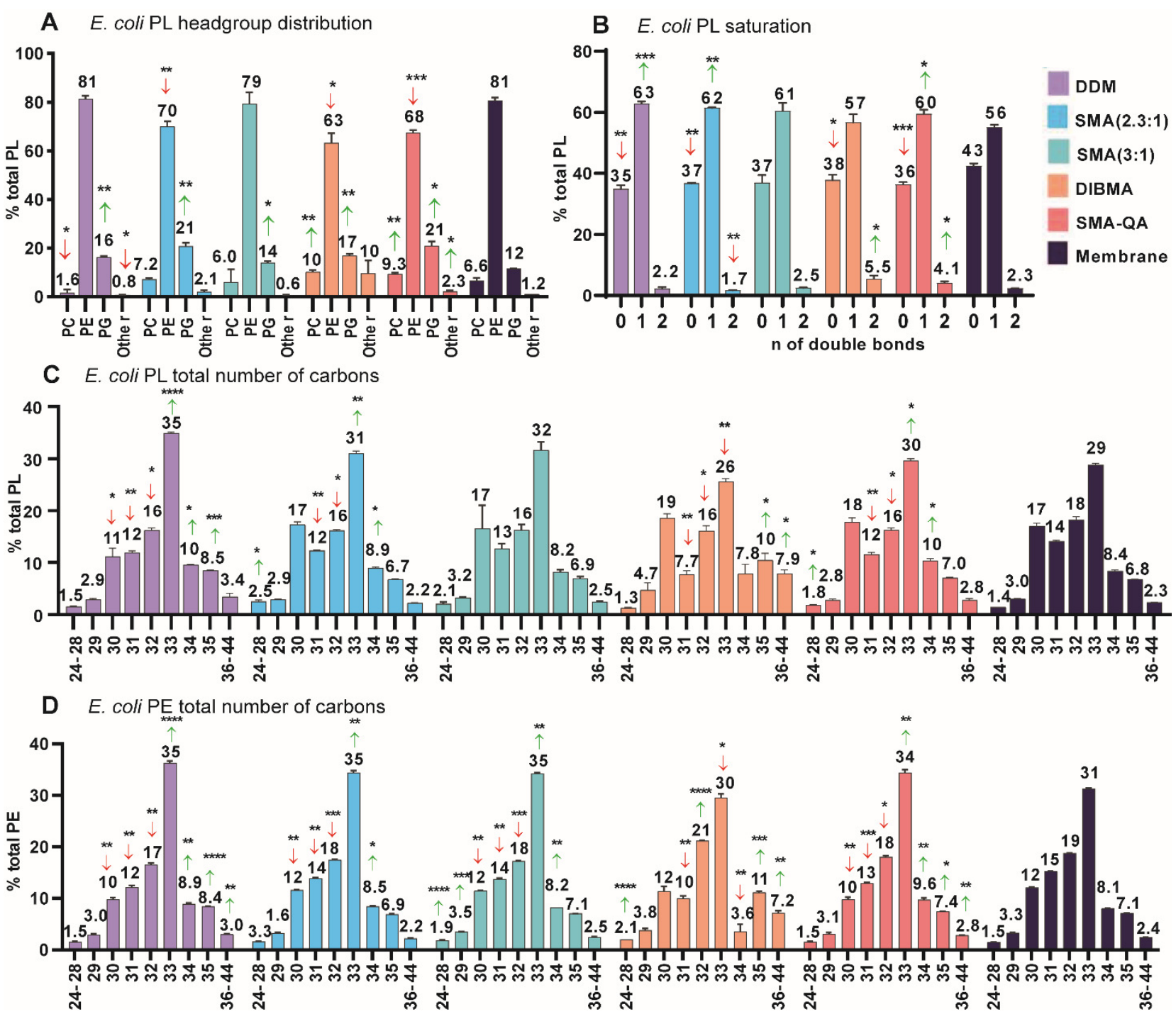

Figure $3 \mathrm{PL}$ content in solubilized and unaltered membrane of $E$. Coli analyzed by LC-MS/MS. (A) Headgroup distribution of PL species. The column "other" includes: PS, PI and PA headgroups. (B) Saturation of PL species. The number of double bonds indicated are those found in the total fatty acid chains of a single PL species. (C) Distribution of carbon atoms present in fatty acid chains of the total PL species. (D) Distribution of carbon atoms present in fatty acid chains of the total PE species. Data points correspond to three technical replicates. Error bars represent \pm S.D. Significant differences (upon one-way ANOVA) are denoted as * $(p$ $<0.05),{ }^{* *}(p<0.01),{ }^{* * *}(p<0.001),{ }^{* * * *}(p<0.001)$. 


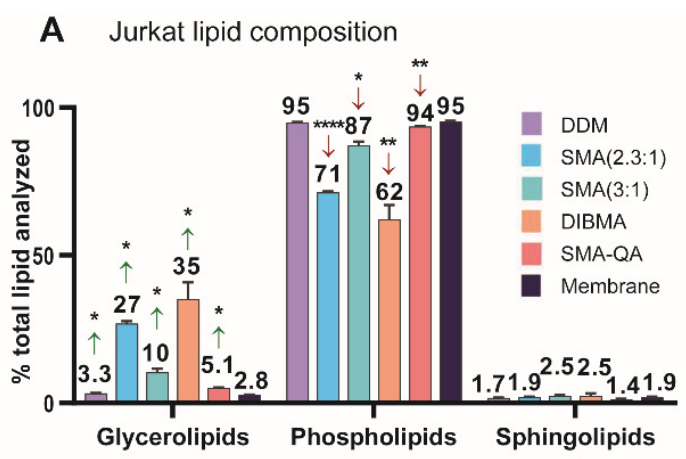

B Jurkat $\mathrm{PL}$ headgroup distribution

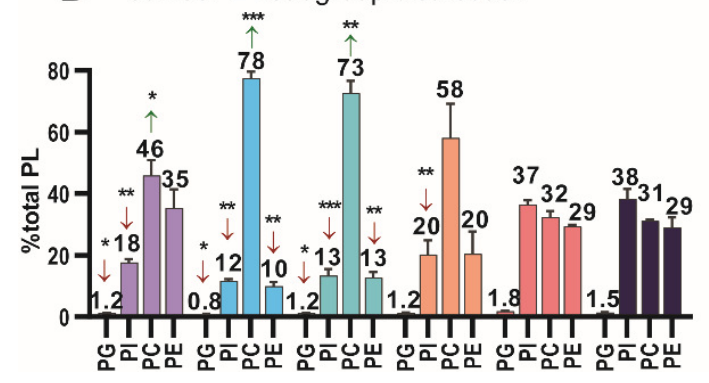

Figure 4. Lipid composition of solubilized membranes and membrane pellet of Jurkat cells analyzed by LC-MS/MS, which allowed for GL, PL and SL identification. (A) Lipid class distribution in each solubilized sample compared with the membrane pellet. (B) Headgroup distribution of PL species. Data points correspond to three technical replicates, except for technical duplicates for SMA-QA. Error bars represent \pm S.D. Significant differences (upon one-way ANOVA) are denoted as ${ }^{*}(p<0.05),{ }^{* *}(p<0.01),{ }^{* * *}(p<0.001),{ }^{* * *}(p<0.001)$. 

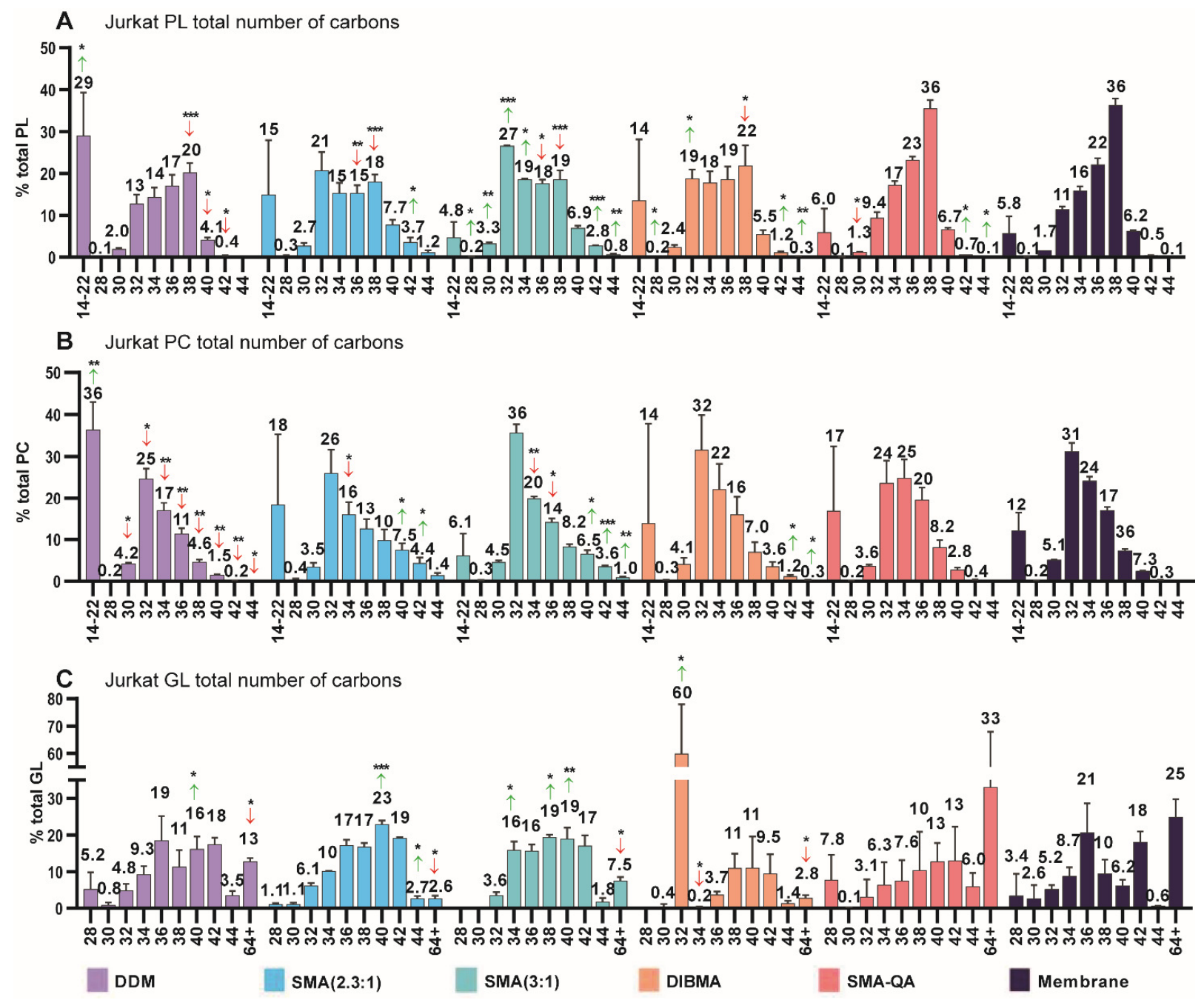

Figure 5 Distribution of carbon atoms present in FA chains of (A) all PL species, (B) PC species and (C) GL species from Jurkat solubilized membranes and membrane pellet. The PC length profiles observed for the solubilized samples largely resemble to that of the membrane, with some differences observed for the detergent sample that was enriched in PC species with 4-6 and 8 or more double bonds and depleted of $1-2$ and 7 double bonds. Contrarily, the unmodified SMA samples were enriched in 6 and more double bond PC species and SMA(2.3:1) depleted of saturated and monounsaturated PC lipids. In general, the differences seen in total PL are therefore a result of the distinct headgroup composition of the samples. Data points correspond to three technical replicates, except for technical duplicates for SMAQA. Error bars represent \pm S.D. Significant differences (upon one-way ANOVA) are denoted as ${ }^{*}(p<0.05),{ }^{* *}(p<0.01),{ }^{* * *}(p<0.001),{ }^{* * * *}(p<0.001)$. 


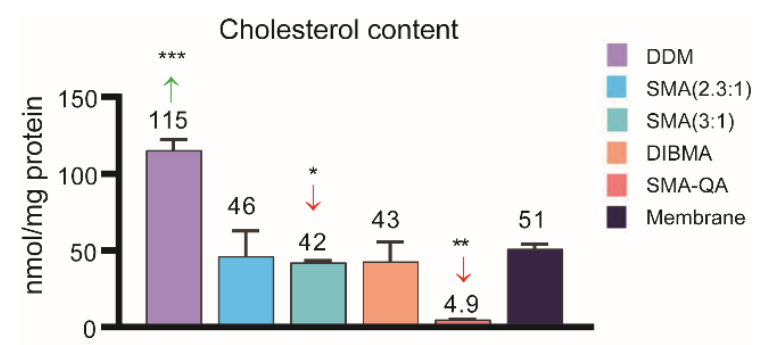

Figure 6 Cholesterol ( $\mathrm{nmol}$ per $\mathrm{mg}$ of protein) in Jurkat membranes and solubilized membranes using Amplex ${ }^{\mathrm{TM}}$ Red Cholesterol Assay Kit (triplicate measurements). Error bars represent \pm S.D. Significant differences compared with the membrane (upon individual t-tests) are denoted as ${ }^{*}(p<0.05),{ }^{* *}(p<0.01),{ }^{* * *}(p<0.001)$. 
Supplemental Information

\title{
Comparative analysis of maleic acid copolymer-based lipid nanodiscs reveals preferential lipid and protein solubilization characteristics
}

\author{
Marta Barniol-Xicota ${ }^{\mathrm{a}}$ and Steven H. L. Verhelst* $*$ a,b \\ ${ }^{a}$ KU Leuven - University of Leuven, Laboratory of Chemical Biology, Department of Cellular \\ and Molecular Medicine, Herestraat 49 box 802, 3000 Leuven, Belgium \\ ${ }^{\mathrm{b}}$ Leibniz Institute for Analytical Sciences ISAS, AG Chemical Proteomics, Otto-Hahn-Str. 6b, \\ 44227 Dortmund, Germany
}

\section{Index}

Materials and methods

$\begin{array}{ll}\text { IR spectra of the xMA polymers } & \text { S8 }\end{array}$

$\begin{array}{ll}\text { Supplemental Figure S1 S11 } & \text { S1 }\end{array}$

$\begin{array}{ll}\text { Supplemental Figure S2 } & \text { S12 }\end{array}$

$\begin{array}{ll}\text { Supplemental Figure S3 } & \text { S13 }\end{array}$

$\begin{array}{ll}\text { Supplemental Figure S4 S13 } & \text { S4 }\end{array}$

$\begin{array}{ll}\text { Supplemental Figure S5 } & \text { S14 }\end{array}$

$\begin{array}{ll}\text { Supplemental Figure S6 } & \text { S15 }\end{array}$

$\begin{array}{ll}\text { Supplemental Figure S7 } & \text { S16 }\end{array}$

$\begin{array}{ll}\text { Supplemental Figure S8 } & \text { S17 }\end{array}$

$\begin{array}{ll}\text { Supplemental Figure S9 } & \text { S18 }\end{array}$

$\begin{array}{ll}\text { Supplementary references } & \text { S19 }\end{array}$ 


\section{Materials and methods}

XIRAN® (SL25010 S25; SL30010 S30; SZ25010) and Sokalan C9 were a kind gift from Polyscope and BASF respectively. All other materials were purchased from commercial vendors and used without prior purification.

\section{Statistical analysis}

Statistical analysis was performed using Prism 8 (GraphPad Software, USA), including multiple unpaired t-tests with Welch correction and one-way Brown-Forsythe Welch ANOVA with Dunett's correction.

\section{Lipid nomenclature}

The lipid nomenclature and classification follow the recommendations of the International Lipid Classification and Nomenclature Committee (LIPID MAPS). ${ }^{1,2}$

\section{Preparation of SMA and DIBMA}

$37 \% \mathrm{HCl}(\sim 7 \mathrm{~mL})$ was slowly added to $20 \mathrm{~mL}$ of Sokalan C9 solution, XIRAN® SL25010 S25 or XIRAN® SL30010 S30, until acidic $\mathrm{pH}$ was reached ( $\mathrm{pH}<2$, checked by $\mathrm{pH}$ paper). The precipitated white solid was washed with $30 \mathrm{~mL}$ of miliQ water and spun down at $4000 \times \mathrm{g}$ for $30 \mathrm{~min}$. The supernatant was discarded and the same wash procedure repeated 3 more times. The wet white solid was snap-frozen in liquid nitrogen and lyophilized to dry powder. From the lyophilized xMA powder, 6\% (w/v) stock solutions in $50 \mathrm{mM}$ HEPES, $0.5 \mathrm{M} \mathrm{NaCl}$ were created. To this end, the $\mathrm{pH}$ was adjusted to 8 by dropwise addition of $\mathrm{NaOH}(10 \mathrm{M}$ at first, later $1 \mathrm{M})$ while sonicating in an ultrasound waterbath to foster the complete dissolution of the xMA. The stock solutions were stored at $-20^{\circ} \mathrm{C}$ in the dark.

\section{Preparation of styrene maleimide quaternary ammonium (SMA-QA)}

SMA-QA was prepared following the reported procedure of Ravula et al. ${ }^{3}$ In short, $770 \mathrm{mg}$ of styrene maleic acid anhydride ratio 2.3:1 and $1 \mathrm{~g}$ of 2-aminoethyltrimethylamonium chloride (5.71 mmol) were dissolved in $23 \mathrm{ml}$ of anhydrous DMF. To the solution, $3.84 \mathrm{~mL}$ of triethylamine $(26.89 \mathrm{mmol})$ were 
added and the whole was heated to $100^{\circ} \mathrm{C}$ under stirring for 20 hours. Once the solution was at room temperature, the product was precipitated using diethyl ether. This precipitated was recovered upon vacuum filtration and washed three times with diethyl ether. The dried intermediate was added to $30 \mathrm{ml}$ acetic anhydride containing $660 \mathrm{mg}$ of sodium acetate and $200 \mathrm{mg}$ of triethyl amine and the whole was heated to $100^{\circ} \mathrm{C}$ overnight. Once the solution was at room temperature, the product was precipitated using diethyl ether and washed three times with this same solvent. The crude product was then dissolved in water and passed through a Sephadex G10 column. The product was lyophilized and characterized using IR. In order to prepare the working SMA-QA stocks, $60 \mathrm{mg}$ of pure product were dissolved in 1 $\mathrm{mL}$ of $50 \mathrm{mM}$ HEPES $0.5 \mathrm{M} \mathrm{NaCl}$ buffer.

\section{Production and solubilization of $E$. coli membranes}

Escherichia coli strain DH5 $\alpha$ were grown in $500 \mathrm{~mL} \mathrm{LB}$ media at $37^{\circ} \mathrm{C}$ for $16 \mathrm{~h}$. The cells were pelleted at $3000 \mathrm{rpm}$ at $4{ }^{\circ} \mathrm{C}$ for $30 \mathrm{~min}$. Pelleted cells were lysed by French Press in lysis buffer (20 mM HEPES, pH 7.4; $0.1 \mathrm{M} \mathrm{NaCl} ; 10 \%$ glycerol; complemented with Roche Complete inhibitor cocktail). The mixture was centrifuged $3,000 \times \mathrm{g}, 15 \mathrm{~min}$ at $4{ }^{\circ} \mathrm{C}$ to remove unbroken cells, and at 100,000 $\times \mathrm{g}, 60$ min, $4{ }^{\circ} \mathrm{C}$ to pellet the membrane fraction. The membrane pellet was resuspended in buffer $50 \mathrm{mM}$ HEPES-NaOH, $\mathrm{pH} 7.8 ; 0.3 \mathrm{M} \mathrm{NaCl}$ to a concentration that will afford $30 \mathrm{mg}$ membrane pellet per $\mathrm{mL}$ of buffer after addition of solubilizing agent. The resuspended pellet was solubilized by addition of 2.5 $\% \mathrm{wt} / \mathrm{v}$ of solubilizing agent from a previously prepared $6 \%$ stock solution. The solubilizing agents used were: styrene-maleic acid copolymer (SMA(3:1) or SMA(2.3:1), respectively), SMA-QA, maleic acid and diisobutylene (2,4,4-trimethylpent-1-ene) (DIBMA) or $\beta$-D-dodecyl-maltoside (DDM). The mixture was left rocking for $2 \mathrm{~h}$ at $37^{\circ} \mathrm{C}$ when using xMA or at $4{ }^{\circ} \mathrm{C}$ when using DDM. Centrifugation at $100,000 \times \mathrm{g}, 40 \mathrm{~min}, 4{ }^{\circ} \mathrm{C}$ removed unsolubilized membranes. The supernatants containing the solubilized membrane were separated from the insoluble pellet and used on further experiments.

\section{Preparation of solubilized Jurkat cell membranes (adapted from ${ }^{4-5}$ )}

Cells $\left(3.4 \times 10^{8}\right)$ were resuspended in $18 \mathrm{~mL}$ of ice-cold hypotonic buffer $(10 \mathrm{mM}$ HEPES pH 7.5, 42 $\mathrm{mM} \mathrm{KCl}, 5 \mathrm{mM} \mathrm{MgCl}$, protease inhibitor mixture), incubated on ice for $15 \mathrm{~min}$. The suspension was 
then passed $10 \times$ through a 26-gauge needle. The supernatant was divided in 18 aliquots of $1 \mathrm{~mL}$ and centrifuged for $20 \mathrm{~min}$ at $25,000 \mathrm{~g}, 2^{\circ} \mathrm{C}$ to pellet the membranes. 3 aliquots were kept separate and each of the remaining 16 aliquots were resuspended to a concentration of $20 \mathrm{mg} / \mathrm{mL}$ in a buffer containing $50 \mathrm{mM}$ HEPES $\mathrm{pH}=7.8,0.3 \mathrm{NaCl}$, benzonase and 2\% solubilizing agent (DDM, SMA(2.3:1), $\operatorname{SMA}(3: 1)$, DIBMA or SMA-QA). The samples were rocked overnight at $37^{\circ} \mathrm{C}$ if using xMA or at $4{ }^{\circ} \mathrm{C}$ if using DDM. After this time the samples were centrifuged for $45 \mathrm{~min}$ at $100,000 \mathrm{~g} 4^{\circ} \mathrm{C}$. The supernatants containing the solubilized membrane were separated from the insoluble pellet and used in further experiments.

\section{Phosphate and protein concentration determination}

Protein concentrations were determined using the Pierce ${ }^{\mathrm{TM}}$ BCA Protein Assay Kit (Thermo Scientific ${ }^{\mathrm{TM}}$ ), according to instructions by the vendor.

For determination of the amount of phospholipids in xMALPs, samples (triplicates; $50 \mu \mathrm{L}$ ) and different amounts of a phosphate standard (0.65 mM; Sigma-Aldrich P3869) were lyophilized to remove solvent. Next, samples were treated with $90 \mu \mathrm{L}$ of $8.9 \mathrm{~N} \mathrm{H}_{2} \mathrm{SO}_{4}$ and heated at $230{ }^{\circ} \mathrm{C}$ for $25 \mathrm{~min}$. After cooling down, $30 \mu \mathrm{L}$ of hydrogen peroxide was added and heating was continued for $30 \mathrm{~min}$ or until the samples were fully transparent. Samples were diluted with $330 \mu \mathrm{L}$ water. Next, $50 \mu \mathrm{L}$ of $2.5 \%$ ammonium molybdate in water (w/v) and $50 \mu \mathrm{L}$ of $10 \%$ ascorbic acid in water (w/v) were added. The mixture was heated at $100{ }^{\circ} \mathrm{C}$ for $10 \mathrm{~min}$ and absorption at $820 \mathrm{~nm}$ was read using a Molecular Devices ID3 platereader. Standard curves $\left(\mathrm{R}^{2}=0.99\right)$ and unknowns were calculated using Microsoft Excel.

\section{Protein precipitation $^{6}$}

In order to prevent smearing of protein bands in SDS-PAGE caused by excess polymer, the proteins present in the solubilized fractions were precipitated to separate them from the polymer. An aliquote of ice-cold sample was mixed with 4 volumes of ice-cold $\mathrm{CH}_{3} \mathrm{OH}$ by vortexing. Next, 1 volume of icecold $\mathrm{CHCl}_{3}$ was added. The sample was mixed by vortexing and then 3 volumes of ice-cold $\mathrm{H}_{2} \mathrm{O}$ were added and mixed the same way. After centrifugation (13000g for $5 \mathrm{~min}$ at $4{ }^{\circ} \mathrm{C}$ ), the top aqueous layer 
was removed carefully not to disrupt the protein layer formed at the interface. An additional 4 volumes of ice-cold $\mathrm{CH}_{3} \mathrm{OH}$ were added and mixed by vortexing and the sample was pelleted by centrifuging for 15 min at $15000 \mathrm{~g}$ at $4{ }^{\circ} \mathrm{C}$. Finally the $\mathrm{CH}_{3} \mathrm{OH}$ was removed and the samples were snap-frozen and lyophilized to eliminate possible traces of solvent. Once dry, the protein pellets were resuspended in $\mathrm{H}_{2} \mathrm{O}$ and the protein concentration was determined using a BCA assay kit (standard curve $\mathrm{R}^{2}=0.99$ ). SDS buffer was added to a sample volume containing $15 \mu \mathrm{g}$ of protein and subjected to SDS-PAGE.

\section{Fourier-transform infrared spectroscopy (FTIR)}

Fourier-transform infrared spectra were recorded on a Bruker Vertex 70 spectrometer spectrophotometer. Direct examination of the products was done by Attentuated Total Reflectance (ATR) using the Bruker ATR platinum setup. The recorded spectra were analyzed by the OPUS software Absorption values are expressed as wavenumbers $\left(\mathrm{cm}^{-1}\right)$; only significant absorption bands are given.

\section{Shotgun lipidomics (E. coli)}

The E. coli shotgun Lipidomics was performed by Lipotype GmbH (Dresden, Germany). The Lipotype Shotgun Lipidomics platform consists of automated extraction of samples, an automated direct sample infusion and high-resolution Orbitrap mass spectrometry including lipid class-specific internal standards to assure absolute quantification of lipids. An in-house developed software - LipotypeXplorer - is used for identification of lipids in the mass spectra.

\section{Equipment and Software}

The equipment and software used in the E. coli lipidomics are: Extraction Hamilton Robotics STARlet; Sample infusion Advion Triversa Nanomate; Mass spectrometry Thermo Scientific Q-Exactive; Lipid Identification LipotypeXplorer; Data processing Lipotype LIMS and LipotypeZoom.

\section{$\underline{\text { Reagents }}$}

Chemicals and solvents of HPLC/LC-MS analytical grade were used.

\section{$\underline{\text { Lipid extraction }}$}


Lipids were extracted using chloroform and methanol. ${ }^{7}$ Samples were spiked with lipid class-specific internal standards prior to extraction. After drying and resuspending in MS acquisition mixture, lipid extracts were subjected to mass spectrometric analysis.

\section{Spectra acquisition}

Mass spectra were acquired on a hybrid quadrupole/Orbitrap mass spectrometer equipped with an automated nano-flow electrospray ion source in both positive and negative ion mode.

Data processing and normalization

Lipid identification using LipotypeXplorer ${ }^{8}$ was performed on unprocessed (*.raw format) mass spectra. For MS-only mode, lipid identification was based on the molecular masses of the intact molecules. MS/MS mode included the collision induced fragmentation of lipid molecules and lipid identification was based on both the intact masses and the masses of the fragments. Prior to normalization and further statistical analysis lipid identifications were filtered according to mass accuracy, occupation threshold, noise and background. Lists of identified lipids and their intensities were stored in a database optimized for the particular structure inherent to lipidomic datasets. Intensity of lipid class-specific internal standards was used for lipid quantification.. 


\section{Shotgun lipidomics (Jurkat)}

\section{Lipid extraction}

$700 \mu \mathrm{l}$ of sample (diluted in water and containing a total of $10 \mu \mathrm{g}$ of protein) was mixed with $800 \mu \mathrm{l} 1$ $\mathrm{N} \mathrm{HCl}: \mathrm{CH}_{3} \mathrm{OH} 1: 8(\mathrm{v} / \mathrm{v}), 900 \mu \mathrm{CHCl}_{3}$ and $200 \mu \mathrm{g} / \mathrm{ml}$ of the antioxidant 2,6-di-tert-butyl-4methylphenol (BHT; Sigma Aldrich). $3 \mu$ of SPLASH® LIPIDOMIX® Mass Spec Standard (\#330707, Avanti Polar Lipids) was spiked into the extract mix. The organic fraction was evaporated using a Savant Speedvac spd111v (Thermo Fisher Scientific) at room temperature and the remaining lipid pellet was stored at $-20^{\circ} \mathrm{C}$ under argon.

\section{Mass spectrometry}

Just before mass spectrometry analysis, lipid pellets were reconstituted in 100\% ethanol. Lipid species were analyzed by liquid chromatography electrospray ionization tandem mass spectrometry (LCESI/MS/MS) on a Nexera X2 UHPLC system (Shimadzu) coupled with hybrid triple quadrupole/linear ion trap mass spectrometer (6500+ QTRAP system; AB SCIEX). Chromatographic separation was performed on a XBridge amide column $(150 \mathrm{~mm} \times 4.6 \mathrm{~mm}, 3.5 \mu \mathrm{m}$; Waters $)$ maintained at $35^{\circ} \mathrm{C}$ using mobile phase A [1 mM ammonium acetate in water-acetonitrile 5:95 (v/v)] and mobile phase B [1 mM ammonium acetate in water-acetonitrile 50:50 (v/v)] in the following gradient: $(0-6 \min : 0 \% \mathrm{~B} \rightarrow 6 \%$ B; 6-10 min: 6\% B $\rightarrow 25 \%$ B; 10-11 min: $25 \%$ B $\rightarrow$ 98\% B; $11-13$ min: $98 \%$ B $\rightarrow 100 \%$ B; 13-19 min: $100 \% \mathrm{~B} ; 19-24 \mathrm{~min}: 0 \% \mathrm{~B}$ ) at a flow rate of $0.7 \mathrm{~mL} / \mathrm{min}$ which was increased to $1.5 \mathrm{~mL} / \mathrm{min}$ from 13 minutes onwards. SM, CE, CER, DCER, HCER, LCER were measured in positive ion mode with a precursor scan of 184.1, 369.4, 264.4, 266.4, 264.4 and 264.4 respectively. TAG, DAG and MAG were measured in positive ion mode with a neutral loss scan for one of the fatty acyl moieties. PC, LPC, PE, LPE, PG, LPG, PI, LPI, PS and LPS were measured in negative ion mode by fatty acyl fragment ions. Lipid quantification was performed by scheduled multiple reactions monitoring (MRM), the transitions being based on the neutral losses or the typical product ions as described above. The instrument parameters were as follows: Curtain Gas $=35$ psi; Collision Gas $=8$ a.u. (medium); IonSpray Voltage $=5500 \mathrm{~V}$ and $-4,500 \mathrm{~V}$; Temperature $=550^{\circ} \mathrm{C}$; Ion Source Gas $1=50 \mathrm{psi}$; Ion Source Gas $2=60 \mathrm{psi}$; Declustering Potential $=60 \mathrm{~V}$ and $-80 \mathrm{~V}$; Entrance Potential $=10 \mathrm{~V}$ and $-10 \mathrm{~V}$; Collision Cell Exit 
Potential $=15 \mathrm{~V}$ and $-15 \mathrm{~V}$. The following fatty acyl moieties were taken into account for the lipidomic analysis: 14:0, 14:1, 16:0, 16:1, 16:2, 18:0, 18:1, 18:2, 18:3, 20:0, 20:1, 20:2, 20:3, 20:4, 20:5, 22:0, 22:1, 22:2, 22:4, 22:5 and 22:6 except for TAGs which considered: 16:0, 16:1, 18:0, 18:1, 18:2, 18:3, $20: 3,20: 4,20: 5,22: 2,22: 3,22: 4,22: 5,22: 6$.

\section{Data Analysis}

Peak integration was performed with the MultiQuant ${ }^{\mathrm{TM}}$ software version 3.0.3. Lipid species signals were corrected for isotopic contributions (calculated with Python Molmass 2019.1.1) and were normalized to internal standard signals. Unpaired T-test p-values and FDR corrected p-values (using the Benjamini/Hochberg procedure) were calculated in Python StatsModels version 0.10.1. 


\section{IR-SPECTRA OF THE XMA POLYMERS}

Styrene maleic acid (2.3:1)

IR (ATR) v: 2927, 1566, 1492, 1411, 759, $698 \mathrm{~cm}^{-1}$.

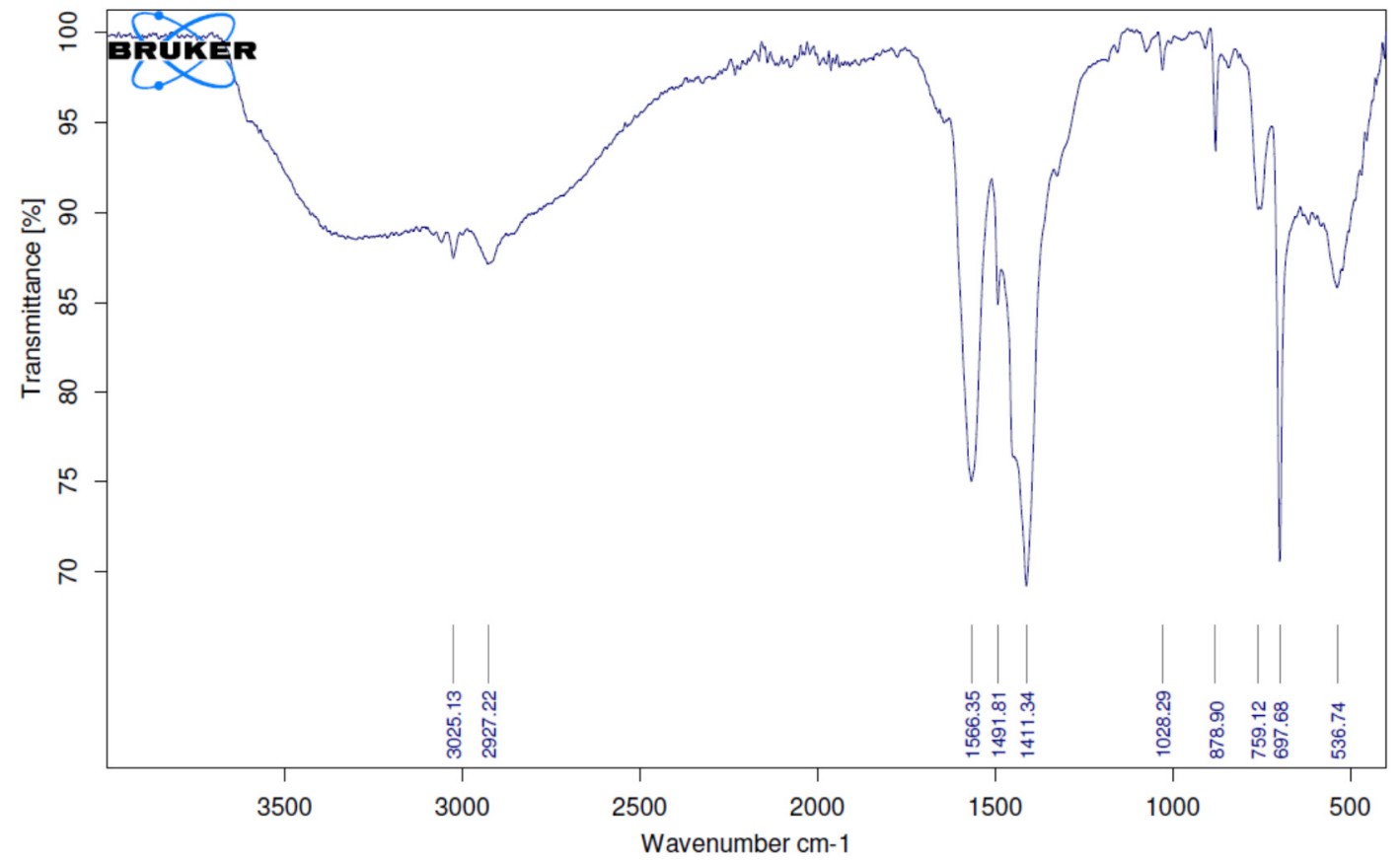

Diisobutylene maleic acid (1:1)

IR (ATR) v: 2947, 1709, 1366, 1186, $921 \mathrm{~cm}^{-1}$.

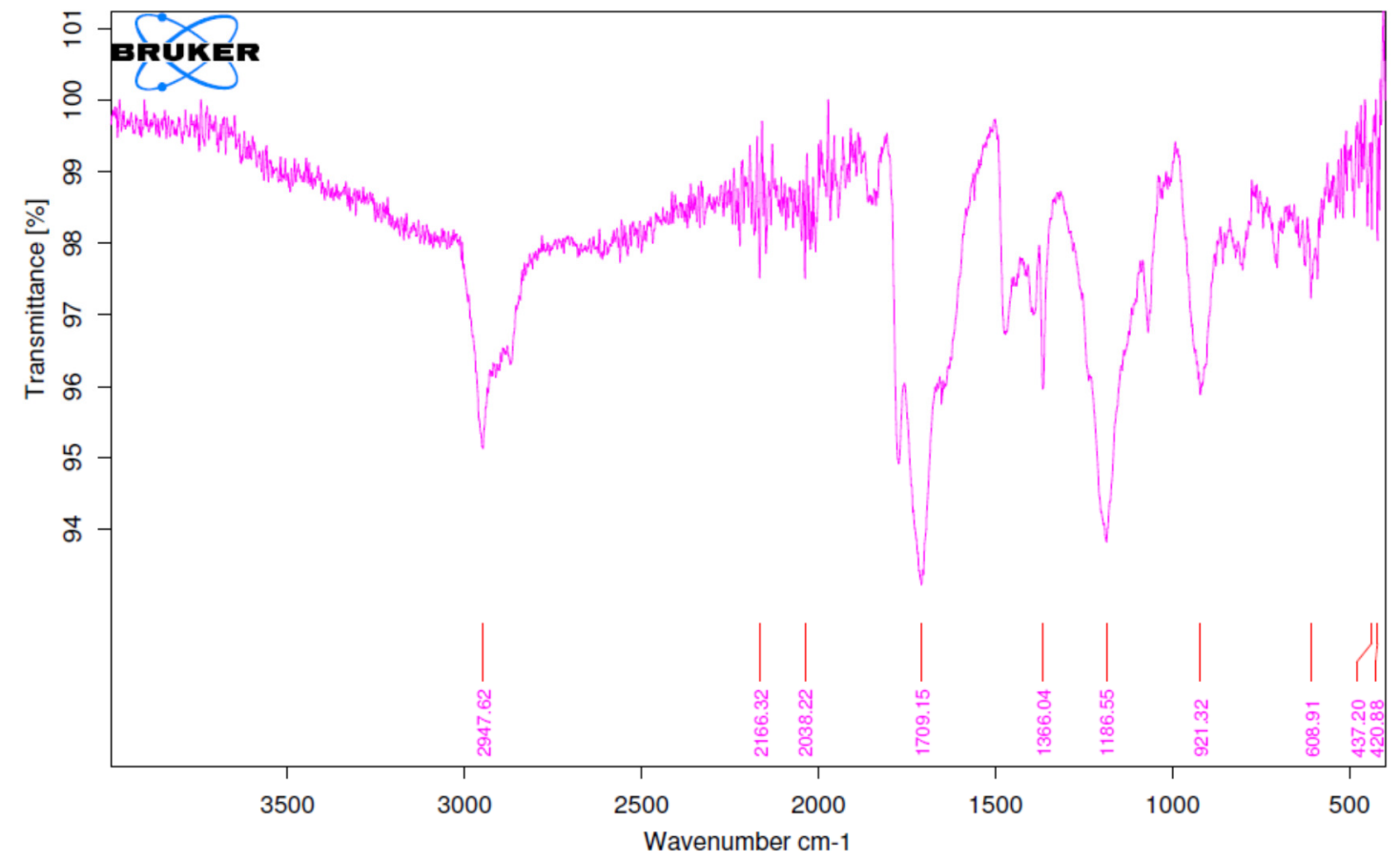


Styrene maleic anhydride (2.3:1)

IR (ATR) v: 2923, 1855, 1774, 1493, 1453, 914, $698 \mathrm{~cm}^{-1}$.

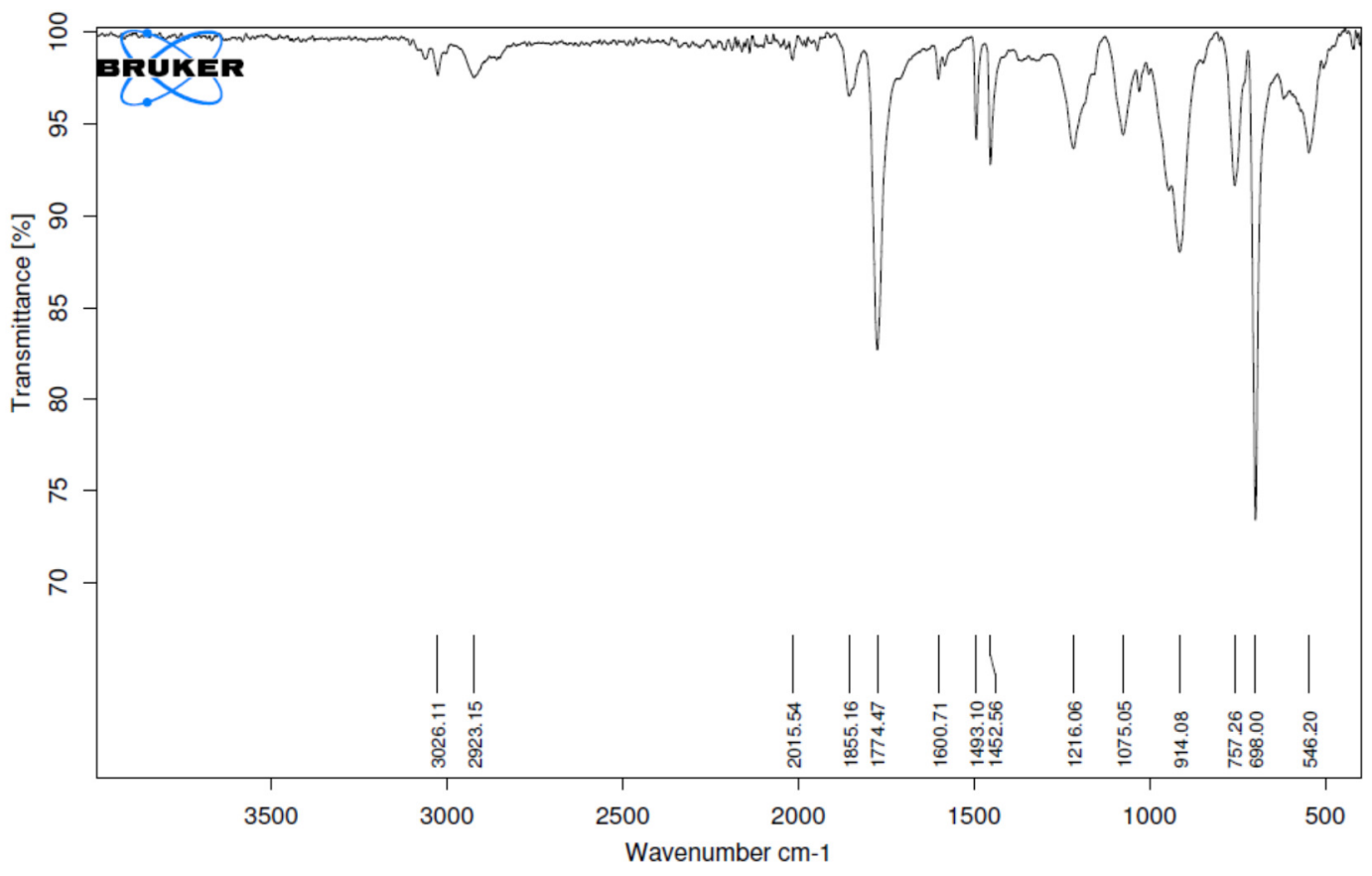

Styrene trimethyl-ethylamime maleimide (SMA-QA)

IR (ATR) v: 3014, 1695, 1486-1146, $701 \mathrm{~cm}^{-1}$.

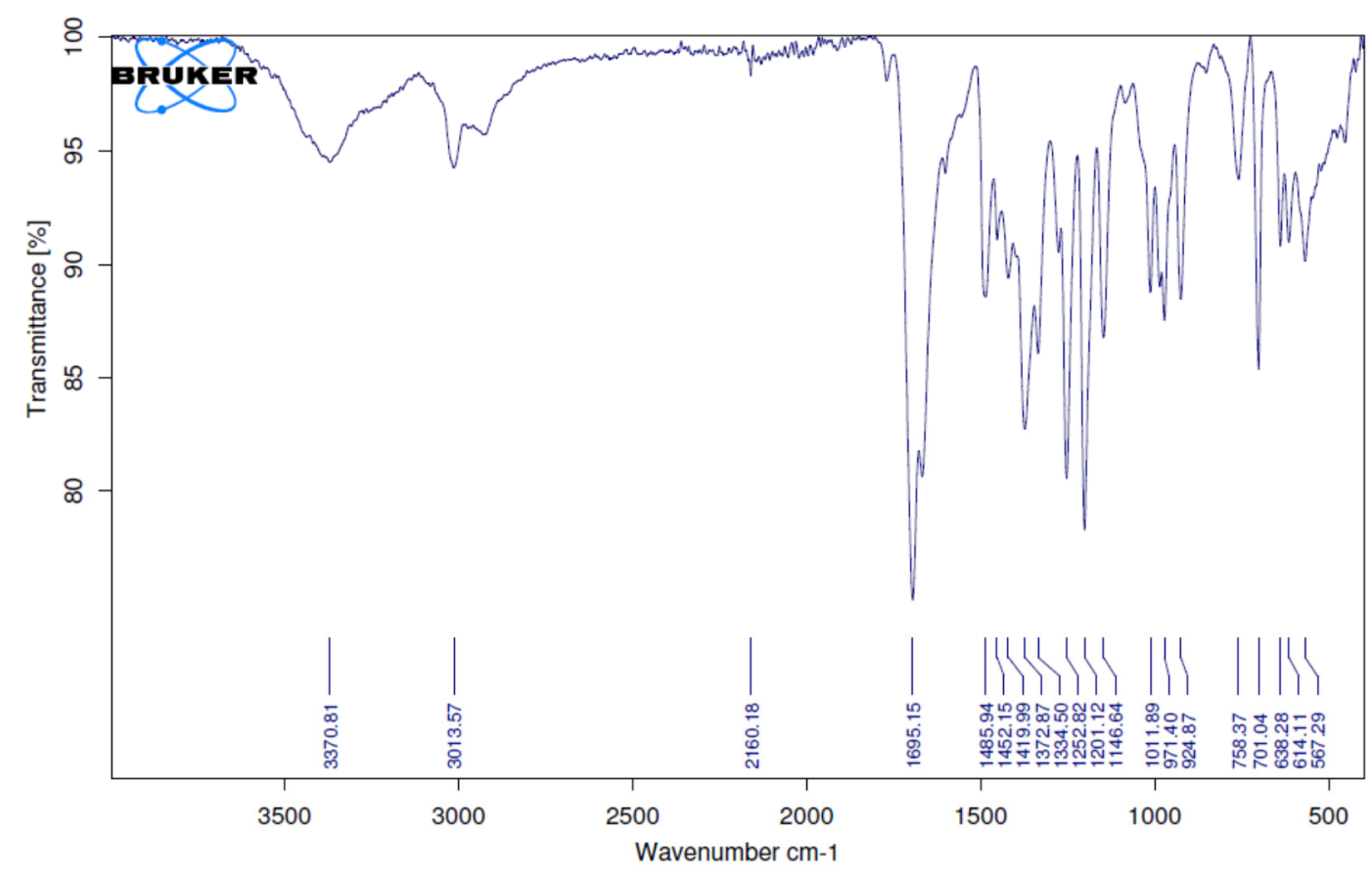


SMA QA vs SMAnh

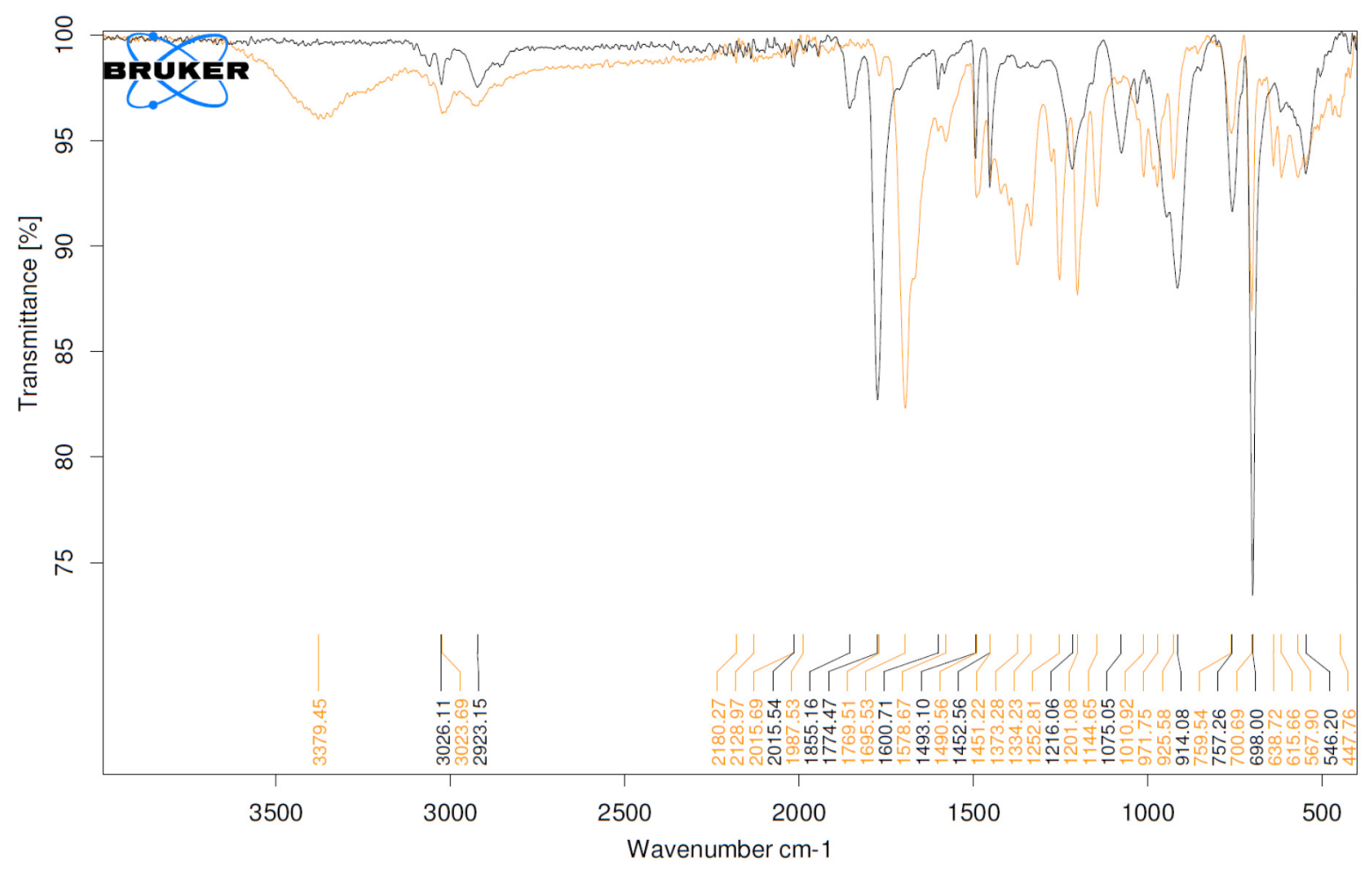

Superimposition of SMA-QA (in orange) and SMAnh IR (in grey) spectra. 
E. coli $\mathrm{PG}$ saturation

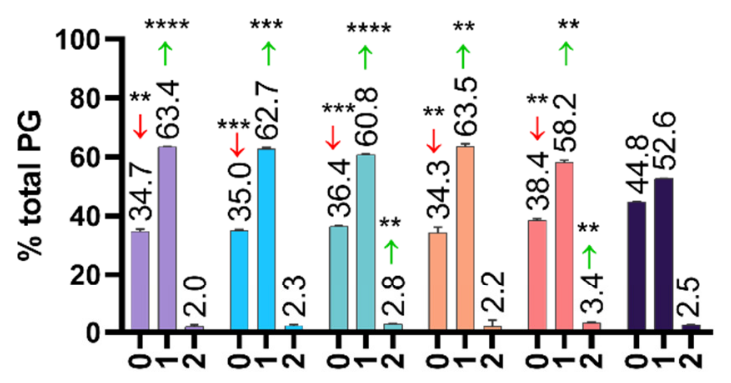

E. coli $\mathrm{PC}$ saturation

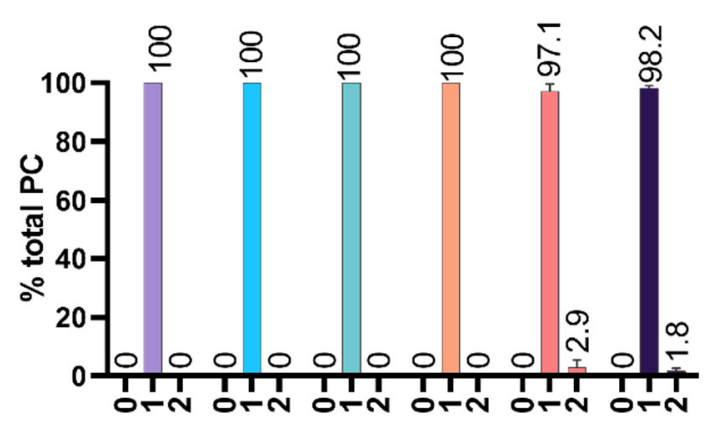

E. coli PS saturation

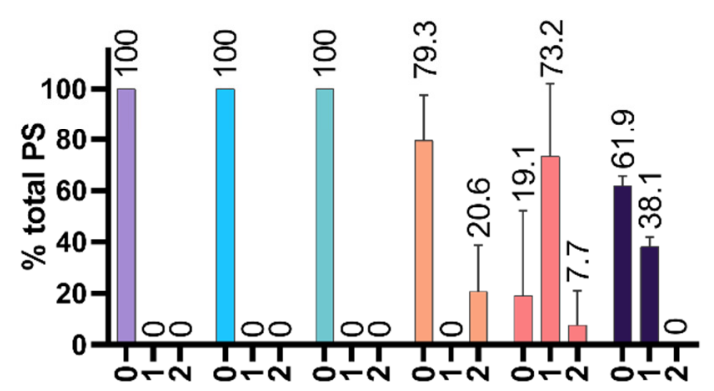

DDM

SMA(2.3:1)

SMA(3:1)

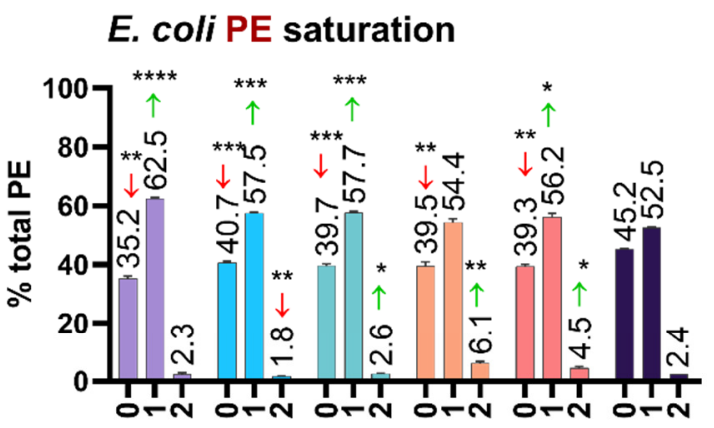

E. coli $\mathrm{PI}$ saturation

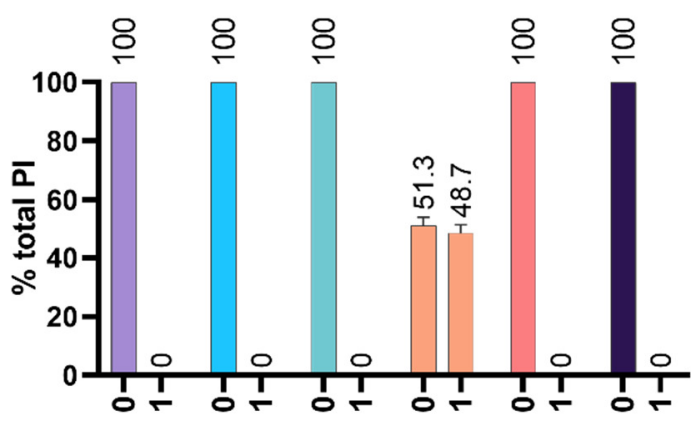

E. coli PA saturation

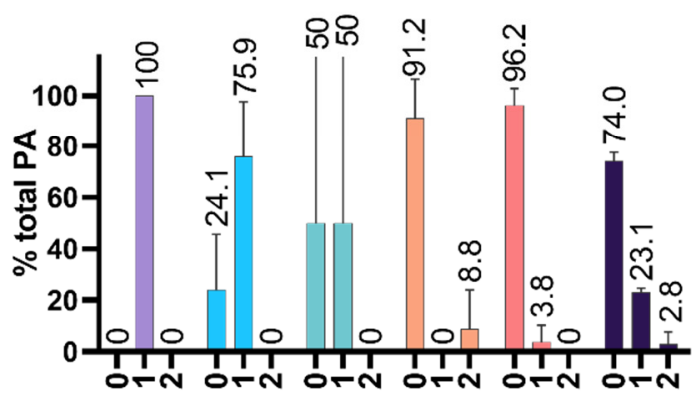

DIBMA SMA-QA

Membrane

Figure S1. Saturation of PL species present in E. coli classified depending on their headgroup, being: PG, PC, PE, PI, PS or PA . The number of double bonds indicated are those found in the total fatty acid chains of a single PL species ${ }^{\ddagger}$. 

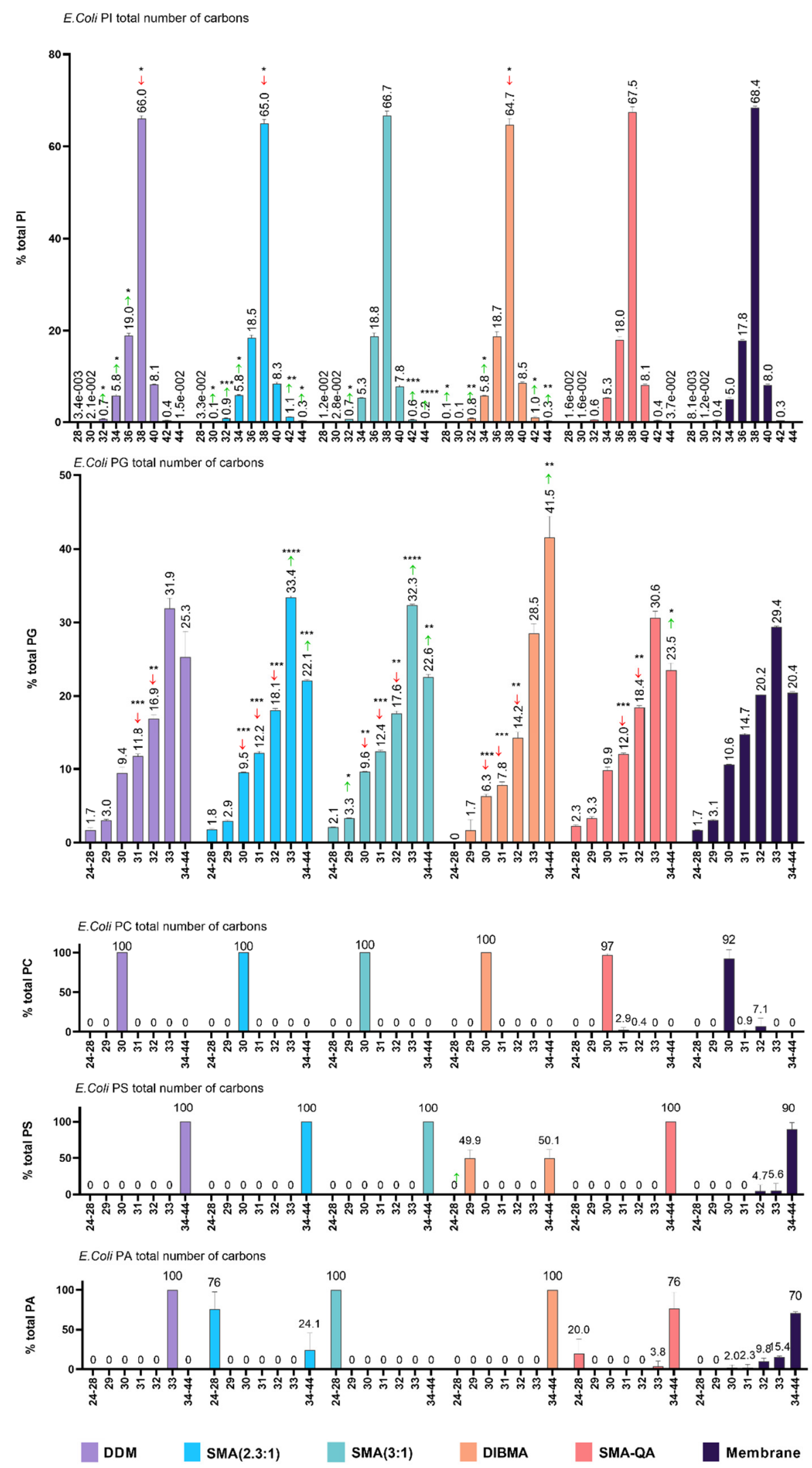

Figure S2. Fatty acid chain length distribution in $E$. coli samples represented by the number of carbons present in the fatty acid chains of the total PI, PG, PC, PS or PA species ${ }^{\ddagger}$. 


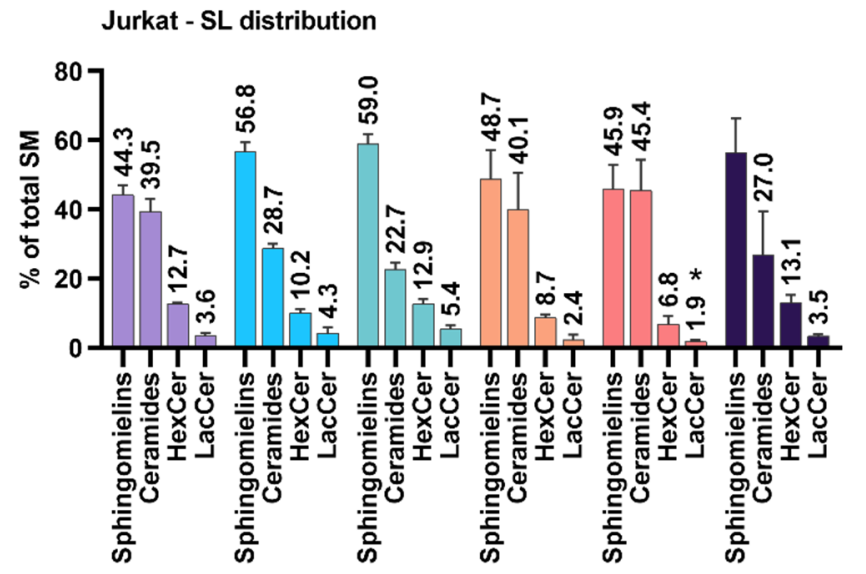

Figure S3. Distribution of species present in the SL fraction of solubilized Jurkat membranes ${ }^{\ddagger}$.

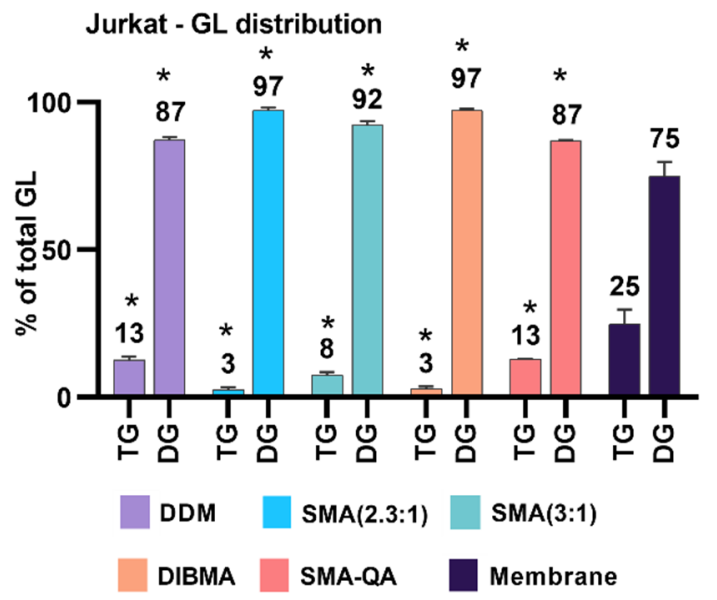

Figure S4. Distribution of species present in the GL fraction of solubilized Jurkat membranes ${ }^{\ddagger}$. DG stands for diacylglycerol and TG for triacylglycerol ${ }^{\ddagger}$. 

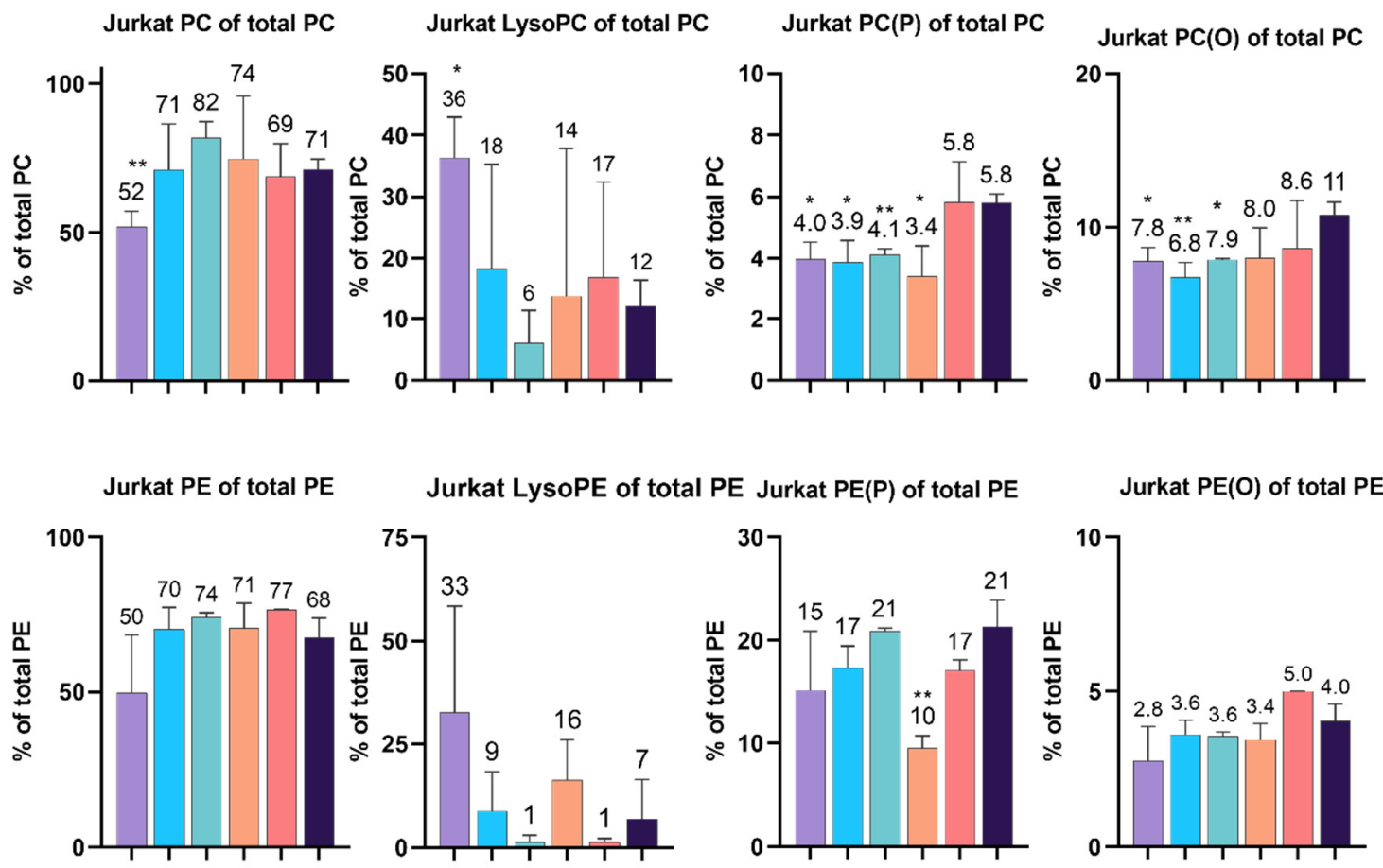

Jurkat LysoPE of total PE Jurkat PE(P) of total PE

Jurkat $P E(O)$ of total $P E$
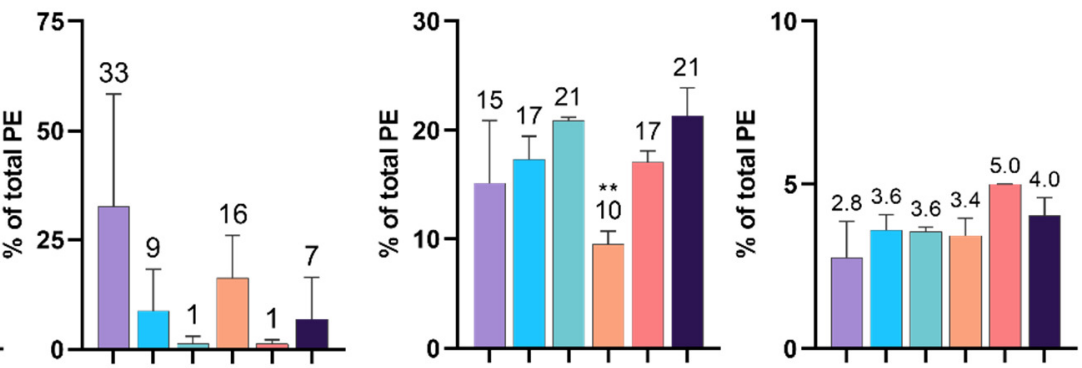

DDM

SMA(2.3:1)

SMA(3:1)

DIBMA

SMA-QA

Membrane

Figure S5. Analysis of the PC and PE fractions of Jurkat membranes. The found different species are: intact PE or PC, being PE or PC with two acyl chains; lysoPE or lysoPC if one of the acyl chain is lost; $P E(P)$ or $P C(P)$ if one of the esther bonds is replaced by an alkenyl ether linkage and $\mathrm{PE}(\mathrm{O})$ or $\mathrm{PC}(\mathrm{O})$ when the esther linkage is replaced by an alkyl ether bond ${ }^{\ddagger}$. 

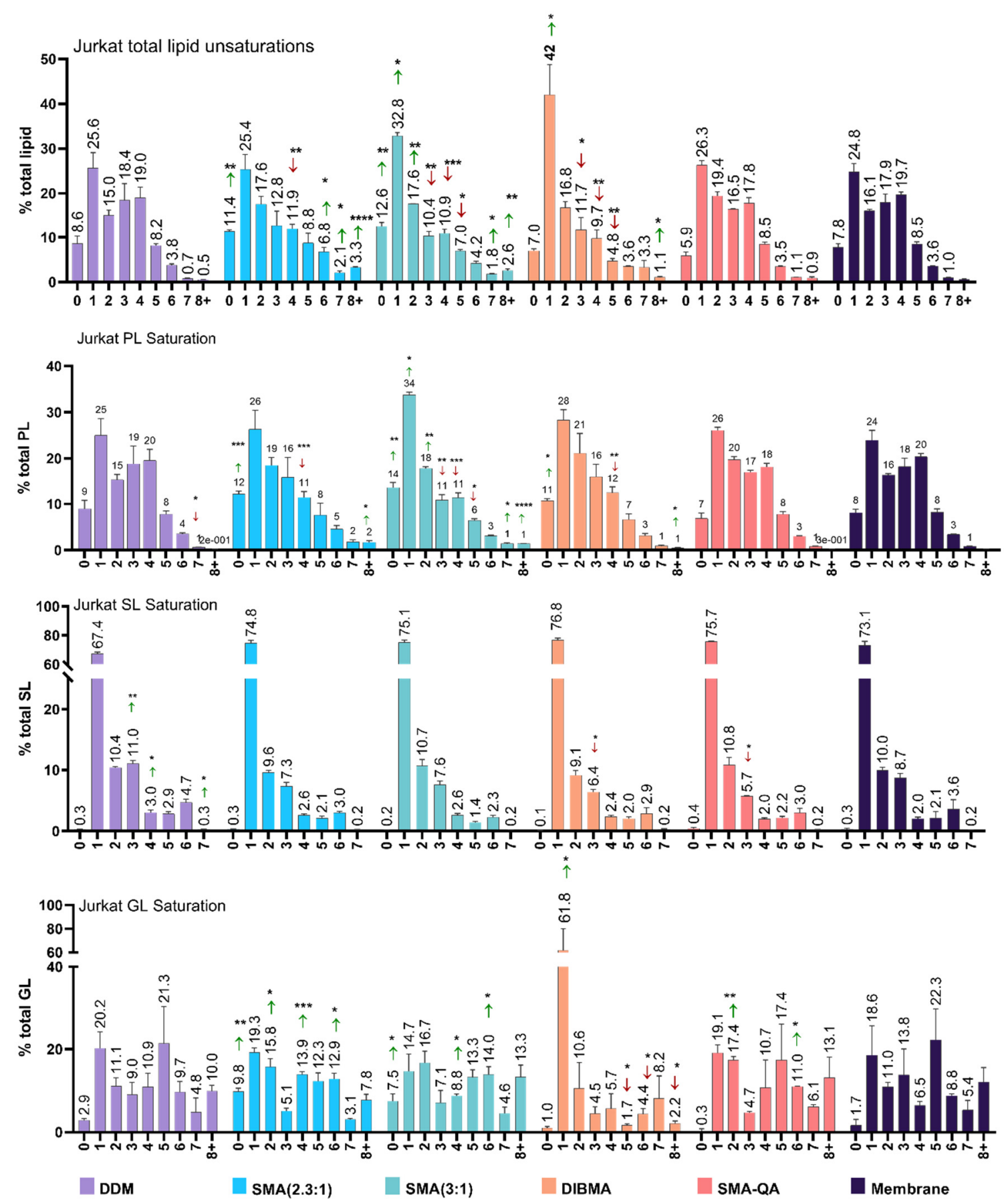

Figure S6. Saturation degree of Jurkat membranes. Represented from top to bottom, saturation profile of: all lipid species; PL; SL and GL ${ }^{\text {. }}$ 

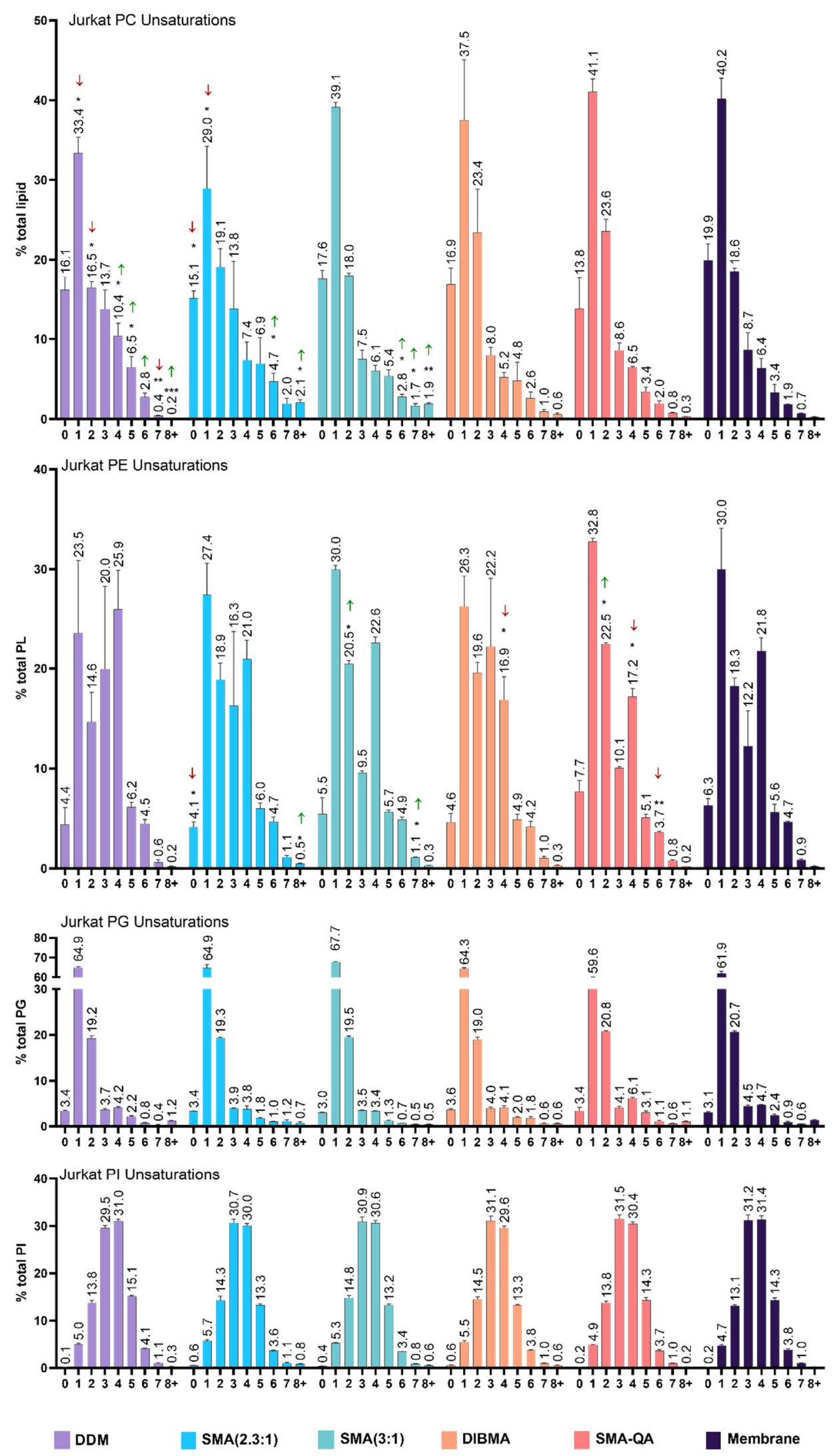

Figure S7. Saturation of PL species present in Jurkat membranes classified depending on their headgroup, being: PC, PG, PE or PI. The number of double bonds indicated are those found in the total fatty acid chains of a single PL species ${ }^{\ddagger}$. 

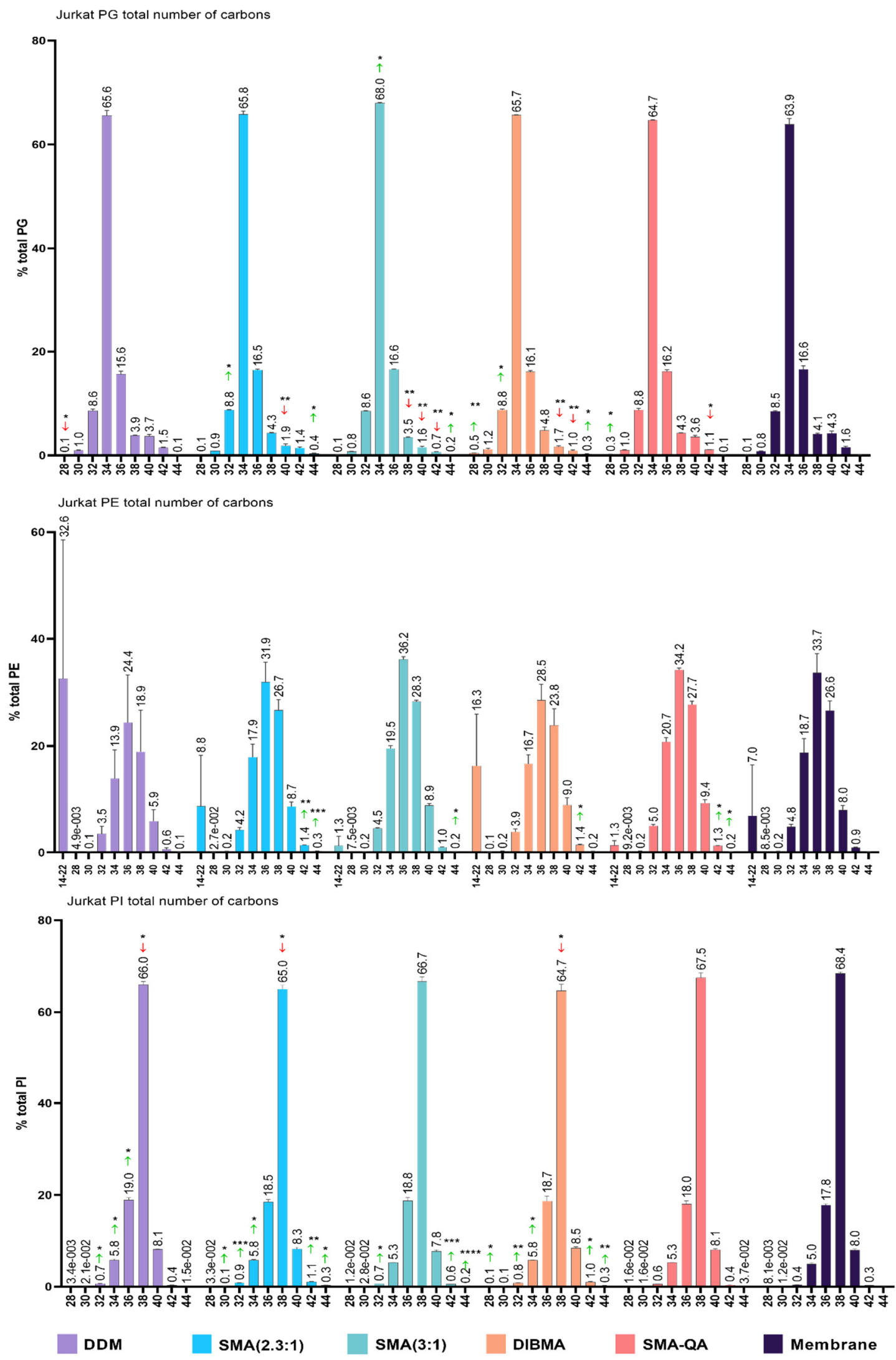

Figure S8. PL fatty acid chain length distribution in Jurkat samples represented by the number of carbons present in the fatty acid chains of the total PG, PE or PI species ${ }^{\ddagger}$. 
Jurkat SL total number of carbons

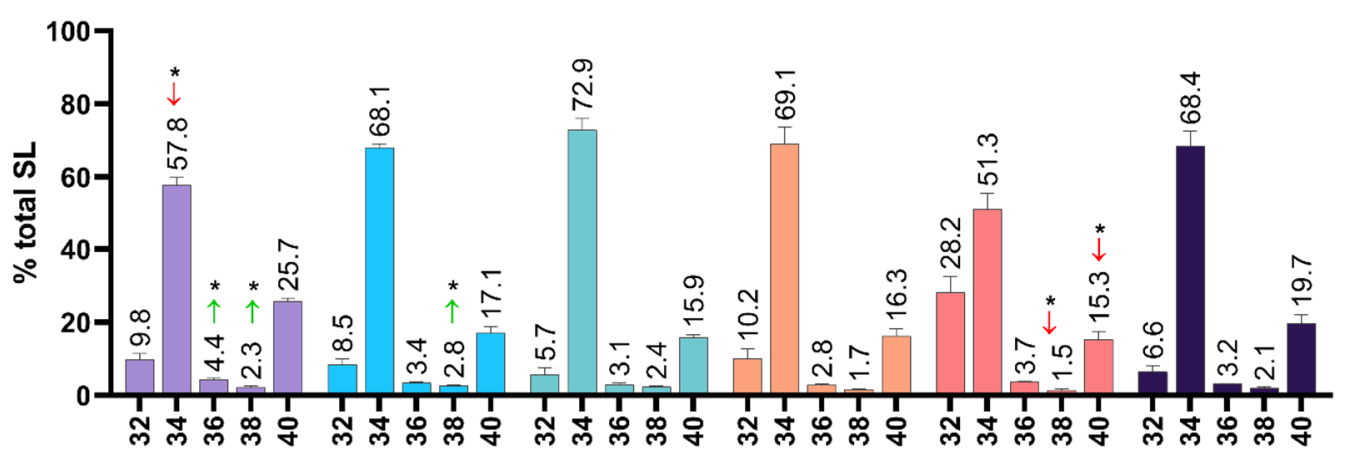

DDM $\quad$ SMA(2.3:1) SMA(3:1) DIBMA $\quad$ SMA-QA

Figure S9. Fatty acid chain length distribution of the SL fraction present in Jurkat samples represented by the number of carbons present in the fatty acid chains ${ }^{\ddagger}$. 


\section{Notes and References}

¥Data points correspond to three technical replicates. Error bars represent \pm S.D. Significant differences (upon one-way ANOVA) are denoted as $*(\mathrm{p}<0.05), * *(\mathrm{p}<0.01), * * *(\mathrm{p}<0.001), * * * *(\mathrm{p}<0.001)$.

1. Fahy, E. et al. A comprehensive classification system for lipids. J. Lipid Res. 46, 839-861 (2005).

2. Fahy, E. et al. Update of the LIPID MAPS comprehensive classification system for lipids. $J$. Lipid Res. 50, 9-14 (2009).

3. Ravula, T., Hardin, N. Z., Ramadugu, S. K., Cox, S. J. \& Ramamoorthy, A. Formation of pHResistant Monodispersed Polymer-Lipid Nanodiscs. Angew. Chemie - Int. Ed. 57, 1342-1345 (2018).

4. Angelisová, P. et al. The use of styrene-maleic acid copolymer (SMA) for studies on T cell membrane rafts. Biochim. Biophys. Acta - Biomembr. 1861, 130-141 (2019).

5. Wessel, D. \& Flügge, U. I. A method for the quantitative recovery of protein in dilute solution in the presence of detergents and lipids. Anal. Biochem. 138, 141-143 (1984).

6. Oluwole, A. O. et al. Solubilization of Membrane Proteins into Functional Lipid-Bilayer Nanodiscs Using a Diisobutylene/Maleic Acid Copolymer. Angew. Chemie - Int. Ed. 56, 19191924 (2017).

7. Sampaio, J. L. et al. Membrane lipidome of an epithelial cell line. Proc. Natl. Acad. Sci. U. S. A. 108, 1903-1907 (2011).

8. Herzog, R. et al. A novel informatics concept for high-throughput shotgun lipidomics based on the molecular fragmentation query language. Genome Biol. 12, 1-25 (2011).

9. Klose, C., Surma, M. A. \& Simons, K. Organellar lipidomics-background and perspectives. Curr. Opin. Cell Biol. 25, 406-413 (2013). 
